\title{
Interface control volume finite element method for modelling multi-phase fluid flow in highly heterogeneous and fractured reservoirs
}

\author{
Ahmad S. Abushaikha ${ }^{\mathrm{a}, \mathrm{b}, *}$, Martin J. Blunt ${ }^{\mathrm{b}}$, Olivier R. Gosselin ${ }^{\mathrm{c}, \mathrm{b}}$, \\ Christopher C. Pain ${ }^{\mathrm{b}}$, Matthew D. Jackson ${ }^{\mathrm{b}}$ \\ a Qatar Environment and Energy Research Institute, Qatar Foundation, PO Box 5825, Doha, Qatar \\ ${ }^{\mathrm{b}}$ Department of Earth Science and Engineering, Imperial College London, London, SW7 2AZ, UK \\ c Total S.A., La Defense 6, 2 Place Jean Millier, 92078 Paris La Defense, France
}

\section{A R T I C L E I N F O}

\section{Article history:}

Received 14 August 2014

Received in revised form 9 February 2015

Accepted 14 May 2015

Available online 21 May 2015

\section{Keywords:}

Control volume finite element method

Mixed hybrid finite element method

Flow in heterogeneous and fractured porous

media

Numerical simulation

Unstructured grid

\begin{abstract}
A B S T R A C T
We present a new control volume finite element method that improves the modelling of multi-phase fluid flow in highly heterogeneous and fractured reservoirs, called the Interface Control Volume Finite Element (ICVFE) method. The method drastically decreases the smearing effects in other CVFE methods, while being mass conservative and numerically consistent. The pressure is computed at the interfaces of elements, and the control volumes are constructed around them, instead of at the elements' vertices. This assures that a control volume straddles, at most, two elements, which decreases the fluid smearing between neighbouring elements when large variations in their material properties are present. Lowest order Raviart-Thomas vectorial basis functions are used for the pressure calculation and first-order Courant basis functions are used to compute fluxes. The method is a combination of Mixed Hybrid Finite Element (MHFE) and CVFE methods. Its accuracy and convergence are tested using three dimensional tetrahedron elements to represent heterogeneous reservoirs. Our new approach is shown to be more accurate than current CVFE methods.
\end{abstract}

(c) 2015 Elsevier Inc. All rights reserved.

\section{Introduction}

Heterogeneous, fractured hydrocarbon reservoirs contain more than half the world's conventional oil [1]. Efficient reservoir management relies on accurate predictions of multi-phase fluid flow in these systems. However, modelling their fluid flow is a challenging task. These reservoirs typically have a complex structure, requiring the use of unstructured grids, with large variations in their material properties. Their permeability fields can vary significantly in space, by orders of magnitude over sharp boundaries, especially in the fractured regions. The Node Control Volume Finite Element (NCVFE) method [2-8] has been traditionally used to model those types of reservoir, Fig. 1 (right). However, since the control volumes are constructed around the nodes and the material properties are assigned on elements, there is a loss of accuracy and associated artificial fluid smearing when modelling multi-phase flow [9-11]. The control volume that is used to conserve the mass

\footnotetext{
* Corresponding author.

E-mail address: aabushaikha@qf.org.qa (A.S. Abushaikha).
} 

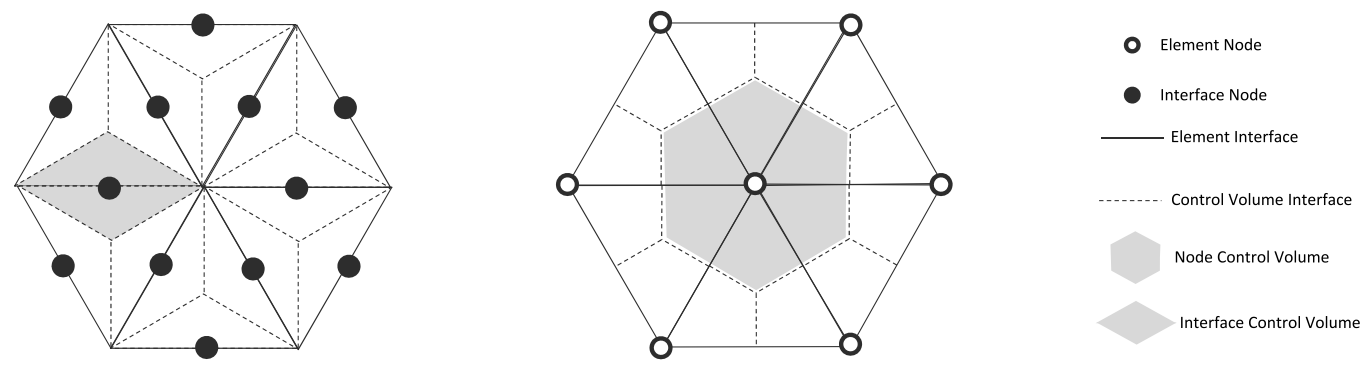

Fig. 1. Interface control volume mesh (left) and node control volume mesh (right) for triangular elements. The material properties are defined on the elements and the pressure and transport variables are computed on the control volumes.

and on which fluid saturation is defined span the different material properties. Thus fluid saturation tends to be smeared across to neighbouring elements, as shown later. Moreover, transport properties such as relative permeability and capillary pressure are saturation dependent, so they are calculated using unphysical values of saturation in the region where the smearing occurs.

The literature addressing the problem of flow between domains of hugely different properties for NCVFE is limited and recent. Nick and Matthai [9] separated the control volumes at element interfaces where material properties vary. Then, they employed the average flux between elements at the interface. Their approach was tested on two-dimensional elements. There was no guarantee of local conservation of mass between the discontinuous control volumes. Bazr-Afkan and Matthai [12] used the elements as control volumes by estimating the inner fluxes between the node control volume sectors using the local mass equation in a post processing step. Then, the fluxes were used to estimate the fluid saturation in the elements. This method is numerically expensive and inconsistent as the number of unknowns, nodes, does not equal the number of elements. Also, it only was tested on two-dimensional elements where the local mass equations are radially constructed, which is not that case for general three-dimensional elements. Verma and Aziz [14] and Edwards [11,15] distributed the material properties on the control volumes which addressed the issue; however this approach decreases the flexibility of the mesh since the geometry of the control volumes is dependent on the primary finite element mesh. Zhang et al. [13] discussed a similar approach to Bazr-Afkan and Matthai in the context of $n$-polygon elements.

In the mixed finite element (MFE) method, the pressure and velocity are solved simultaneously and the fluxes are continuous across element interfaces [16-20]. A secondary grid is not needed as the control volume is the element. Durlofsky [19] compared the MFE and NCVFE methods and showed that the MFE method approximates the solutions more accurately than NCVFE in small highly heterogeneous permeability cases. Huber and Helmig [5] studied the saturation profiles for both methods in heterogeneous media and showed that the mixed method produces sharper less diffusive fronts. In the NCVFE method, the stiffness matrix conductivity coefficients between neighbouring elements are weighted arithmetically, while for MFE they are weighted by taking the harmonic mean. This tends to overestimate the effective conductivity for the NCVFE method and underestimate them for MFE in heterogeneous models [21]. However, for sufficiently large heterogeneous models the deviation from theoretical solutions for both methods is similar, as shown by Lachassagne et al. [22].

The indefinite characteristic of the stiffness matrix of MFE method can be avoided by the hybridisation process of the mixed hybrid finite element (MHFE) method [23-25]. In the hybrid method, the system is solved at the interfaces of elements which more than doubles the number of unknowns for a mesh composed of tetrahedrons. This leads to numerical inconsistency as the number of control volumes, elements, does not equal the number of unknowns, interfaces.

In this work, we suggest a numerical scheme that uses the advantages of both methods, MHFE and CVFE. The scheme is called the Interface Control Volume Finite Element (ICVFE) method. The method calculates the pressure at the interface of elements, instead of at nodes, and constructs the control volumes around them, Fig. 1 (left). Each control volume is shared by, at most, two elements thus decreasing unnecessary fluid smearing. Lowest-order Raviart-Thomas [26] vectorial basis functions (RT0) are used to discretise the pressure equation, similar to the MHFE method [24,25]. Pressure derivatives are calculated using first-order Courant basis functions [27,28], similar to the NCVFE method [2-8]. The fluxes are calculated on interface control volumes according to the properties of the shared elements and the pressure derivative. The method is numerically consistent and mass conservative. However, it is numerically expensive as the degrees of freedom, defined at interfaces, can be more than nine times the number of nodes in a mesh composed of tetrahedron elements. Nevertheless, as shown later, it displays better convergence than NCVFE with similar degrees of freedom.

The paper is organised as follows. In Section 2, the governing equations are introduced. In Section 3, the Interface Control Volume Finite Element method is described, and in Section 4, numerical tests are presented. Finally, conclusions are given in Section 5. 


\section{Governing equations}

We consider two-phase immiscible fluid flow of water and oil in heterogeneous porous media described by the continuity equation and Darcy's law [29]. We assume a slightly compressible rock. The mass balance for the fluid phase $\alpha$ is,

$$
\frac{\partial\left(\phi \rho_{\alpha} S_{\alpha}\right)}{\partial t}=-\nabla \cdot\left(\rho_{\alpha} v_{\alpha}\right)+\rho_{\alpha} q_{\alpha}
$$

$S$ is the saturation of the phase, $\phi$ is the porosity of the rock, $\rho$ is the density of the phase, $q$ is the source-sink rate of the phase, $v$ is the Darcy velocity for phase, and $t$ is time. We also assume capillarity and gravity forces are negligible. The Darcy phase velocity is,

$$
v_{\alpha}=-\lambda_{\alpha} K \nabla P_{\alpha}
$$

where $P$ is the phase pressure, $K$ is the absolute rock permeability, and $\lambda$ is the phase mobility,

$$
\lambda_{\alpha}=\frac{k_{r \alpha}\left(S_{\alpha}\right)}{\mu_{\alpha}}
$$

where $k_{r}$ is the relative permeability of phase and $\mu$ is fluid viscosity of phase. The fluid viscosity is constant and usually different for each phase. The relative permeability is saturation dependent, and in this paper we use Corey-type [30] functions,

$$
k_{r o}=\left(1-S_{w o}\right)^{N_{o}}, \quad k_{r w}=k_{r w}^{o} S_{w o}^{N_{w}}, \quad S_{w o}=\frac{S_{w}-S_{w_{i}}}{1-S_{w_{i}}-S_{o r}}
$$

where $N_{o}$ and $N_{w}$ are history matching parameters, $k_{r w}^{o}$ is the end point of the water relative permeability, $S_{w o}$ is the normalised water saturation, $S_{w_{i}}$ is initial water saturation and $S_{\text {or }}$ is residual oil saturation.

For reservoir simulation, the domain is assumed to be closed and initial and boundary conditions are known. Wells are represented by source/sink terms. If we assume incompressible fluids the continuity equations for the phases, Eq. (1), are combined to form a pressure equation without the explicit representation of time derivatives of saturations [4]. The pressure equation is,

$$
\phi C_{r} \frac{\partial P}{\partial t}+\nabla \cdot v=q_{t}
$$

where $C_{r}$ is rock compressibility, $q_{t}$ is the total source-sink rate of both phases and $v$ is the total velocity,

$$
v=-\lambda_{t} K \nabla P
$$

where $\lambda_{t}$ is the total mobility given by,

$$
\lambda_{t}=\frac{k_{r o}}{\mu_{0}}+\frac{k_{r w}}{\mu_{w}}
$$

Once the pressure field is solved, Eq. (5), the phase velocities are calculated using Darcy's law, Eq. (2), and the advection and transport of fluid is calculated using Eq. (1). The nonlinear coupling between the pressure and advection equations is by the total mobility, Eq. (7), which is saturation dependent over time and space. Next, we describe the proposed Interface Control Volume Finite Element method.

\section{Interface control volume finite element method (ICVFE)}

We have developed a numerical scheme, ICVFE, which is a combination of the MHFE and CVFE methods. It approximates the pressure and advection equations in space and time using discrete number of degrees of freedom, at the interfaces of elements. It solves a multi-phase flow problem in three steps. First, the pressure is calculated at the interfaces of elements using RT0 element vectorial basis functions under the same assumptions as the MHFE method. Then, the pressure drop needed for the velocity calculation is solved using first-order Courant basis functions. The method is conservative and numerically consistent. Finally, the control volumes are imposed around element interfaces and the fluxes needed for fluid advection are computed using the properties of elements sharing the control volume, Fig. 1 (left). This type of control volume minimises the smearing effect during fluid transport in highly heterogeneous and fractured reservoirs, as we will show in Section 4. In this paper, we only consider three-dimensional tetrahedron elements.

\subsection{Mixed hybrid finite element method}

In this section, we discuss how the pressure and velocity equations are discretised and the assumptions taken to calculate the pressure at interfaces. It is based on the MHFE method [24] where the pressure and the velocity fields are solved simultaneously, and the pressure and fluxes at element's interfaces are assumed continuous, 


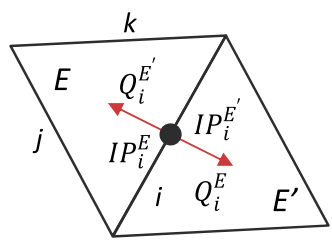

Fig. 2. Two triangle elements $E$ and $E^{\prime}$ sharing interface $i$; where continuity of pressure and fluxes are imposed by MHFE method.

$$
\begin{aligned}
& I P_{A}^{E}=I P_{A}^{E^{\prime}} \\
& Q_{A}^{E}+Q_{A}^{E^{\prime}}=0
\end{aligned}
$$

where $I P$ is the pressure at the centroid of element interface, $Q$ is the interface flux, $E$ is the element number, $A$ is the interface, see Fig. 2.

First, we discretise the velocity equation using the lowest-order Raviart-Thomas vectorial basis functions (RT0). Then, we use it for the pressure equation to approximate the pressures at interfaces.

\subsubsection{The velocity equation}

The lowest-order Raviart-Thomas vectorial basis functions (RT0) are used to approximate the solution of the velocity vector over an element. For a tetrahedron element, they define the velocity by,

$$
\mathbf{v}^{E}=\sum_{i=1}^{4} \mathbf{w}_{i}^{E} Q_{i}^{E}
$$

where $\mathbf{v}^{E}$ is the approximate solution of velocity vector in element $E, \mathbf{w}_{i}^{E}$ is the vectorial basis function and corresponds to a vector having a flux of one at interface $i$ of element $E$ and zero at the other interfaces. It is given by,

$$
\int_{A_{j}^{E}} \mathbf{w}_{i}^{E} \cdot \mathbf{n}_{j}^{E}=\left\{\begin{array}{ll}
1 & \text { if } i=j \\
0 & \text { if } i \neq j
\end{array} \quad \forall j=1, \ldots, 4\right.
$$

where $\mathbf{n}_{j}^{E}$ is the normal outward oriented vector and $A_{j}^{E}$ is the area of interface $j$ of element $E$. Eq. (11) can be written as,

$$
\mathbf{w}_{i}^{E} \cdot \mathbf{n}_{j}^{E}=\left\{\begin{array}{ll}
\frac{1}{A_{j}^{E}} & \text { if } i=j \\
0 & \text { if } i \neq j
\end{array} \quad \forall j=1, . ., 4\right.
$$

Another property of RT0 is $\nabla \cdot \mathbf{w}_{i}^{E}$ is constant over element $E$,

$$
\int_{V^{E}} \nabla \cdot \mathbf{w}_{i}^{E}=1
$$

where $V^{E}$ is volume of element $E$, and Eq. (13) can be written as,

$$
\nabla \cdot \mathbf{w}_{i}^{E}=\frac{1}{V^{E}}
$$

Multiplying the total velocity equation, Eq. (6), by $\mathbf{w}_{i}^{E}$, then integrating over the volume of element $E$ and using Gauss's theorem yields,

$$
\int_{V^{E}} \mathbf{v}^{E} \cdot \mathbf{w}_{i}^{E}=-\lambda_{t}^{E} K^{E} \int_{V^{E}} \nabla P^{E} \cdot \mathbf{w}_{i}^{E}=\lambda_{t}^{E} K^{E}\left(\int_{V^{E}} P^{E} \nabla \cdot \mathbf{w}_{i}^{E}-\sum_{j=1}^{4} \int_{A_{j}^{E}} I P_{j}^{E} \mathbf{w}_{i}^{E} \cdot \mathbf{n}_{j}^{E}\right) \quad \forall i=1, . ., 4
$$

where $P^{E}$ is the average element pressure and $I P_{j}^{E}$ is interface pressure $j$ of element $E$, see Fig. 3. Using the properties of $\mathbf{w}_{i}^{E}$, Eqs. (12) and (14), and including Eq. (10) in Eq. (15) gives,

$$
\sum_{j=1}^{4} Q_{j}^{E} \int_{V^{E}} \mathbf{w}_{j}^{E} \cdot \mathbf{w}_{i}^{E}=\lambda_{t}^{E} K^{E}\left(P^{E}-I P_{i}^{E}\right) \quad \forall i=1, . ., 4
$$



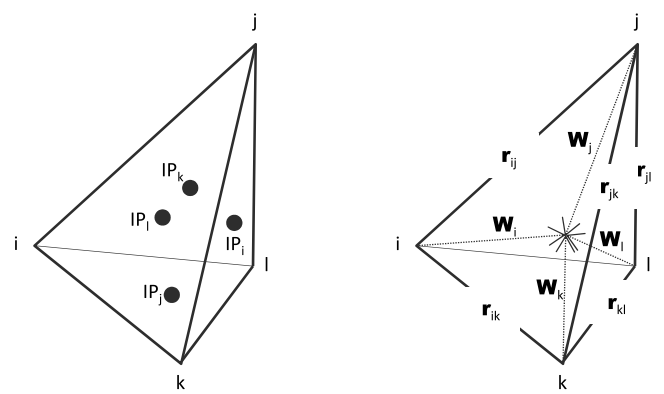

Fig. 3. Interface pressures are at the centroid of interfaces for tetrahedron element (left) and the basis functions for the tetrahedron in MHFE method (right).

We can write Eq. (16) in the matrix form which leads to the consistency equation below for tetrahedron elements,

$$
\mathbf{B}^{E}\left[\begin{array}{c}
Q_{i}^{E} \\
Q_{j}^{E} \\
Q_{k}^{E} \\
Q_{l}^{E}
\end{array}\right]=\lambda_{t}^{E} K^{E}\left[\begin{array}{c}
P^{E}-I P_{i}^{E} \\
P^{E}-I P_{j}^{E} \\
P^{E}-I P_{k}^{E} \\
P^{E}-I P_{l}^{E}
\end{array}\right]
$$

where $\mathbf{B}^{E}$ is the elementary $4 \times 4$ symmetric matrix of a tetrahedron element and its values are calculated by,

$$
B_{i, j}^{E}=\int_{V^{E}} \mathbf{w}_{i}^{E} \cdot \mathbf{w}_{j}^{E}
$$

where $\mathbf{w}_{i}^{E}$ for a tetrahedron element $[26,31,32]$ are defined by,

$$
\mathbf{w}_{i}^{E}=\frac{1}{3 V^{E}}\left[\begin{array}{c}
x-x_{i}^{E} \\
y-y_{i}^{E} \\
z-z_{i}^{E}
\end{array}\right]
$$

where $\left(x_{i}^{E}, y_{i}^{E}, z_{i}^{E}\right)$ is coordinates of node $i$ for tetrahedron element $E$.

By integrating Eq. (18) over element volume, the values of the symmetric matrix $\mathbf{B}^{E}$ are given by,

$$
\begin{aligned}
& B_{i, i}=\frac{1}{360 V^{E}}\left[8\left(l_{i j}+l_{i k}+l_{i l}\right)-2\left(l_{j k}+l_{j l}+l_{k l}\right)\right] \quad i, j, k, l \text { all different } \\
& B_{i, j}=\frac{1}{360 V^{E}}\left[12 l_{i j}+3\left(l_{i k}+l_{i l}+l_{j k}+l_{j l}\right)-2 l_{k l}\right] \quad i, j, k, l \text { all different }
\end{aligned}
$$

where $l_{i j}$ is square length $\left\|\mathbf{r}_{i j}\right\|^{2}$ and $\mathbf{r}_{i j}$ is the edge vector from node $i$ to node $j$, see Fig. 3 .

\subsubsection{The pressure equation}

We use the implicit method for the time discretisation of the pressure equation, Eq. (5), and integrate over the volume of element $E$ and employ Eqs. (10) and (14),

$$
V_{p}^{E^{n}} C_{r}^{E} \frac{P^{E^{n+1}}-P^{E^{n}}}{\Delta t}+\sum_{i=1}^{4} Q_{i}^{E^{n+1}}=F_{t}^{E^{n+1}}
$$

where $n$ is time step number, $\Delta t$ is time step value, $F_{t}^{E}$ is the total source/sink volumetric rate term of element $E$, and $V_{p}^{E}$ is the pore volume of element $E$,

$$
V_{p}^{E^{n}}=V^{E} \phi_{\text {initial }}^{E} e^{C_{r}^{E}\left(P^{E^{n}}-P_{\text {initial }}^{E}\right)}
$$

where intial is initial reservoir condition, and we assume a constant rock compressibility. We express $Q_{A}^{E}$ from the consistency equation, Eq. (17),

$$
Q_{A}^{E^{n+1}}=\lambda_{t}^{E^{n}} K^{E}\left(L_{A}^{E} P^{E^{n+1}}-\sum_{A^{\prime} \subset \partial E} B_{A, A^{\prime}}^{E^{-1}} I P_{A^{\prime}}^{n+1}\right)
$$


where $L_{A}^{E}$ is dimensionless shape factor associated with $\mathbf{B}^{E}$,

$$
L_{A}^{E}=\sum_{A^{\prime} \subset \partial E} B_{A, A^{\prime}}^{E^{-1}}
$$

where subscripts $A$ and $A^{\prime}$ are matrix row and column respectively. We put Eq. (24) into Eq. (22) and yield,

$$
V_{p}^{E^{n}} C_{r}^{E} \frac{P^{E^{n+1}}-P^{E^{n}}}{\Delta t}+\lambda_{t}^{E^{n}} K^{E}\left(L^{E} P^{E^{n+1}}-\sum_{A \subset \partial E} L_{A}^{E} I P_{A}^{n+1}\right)=F_{t}^{E^{n+1}}
$$

where

$$
L^{E}=\sum_{A \subset \partial E} L_{A}^{E}
$$

\subsubsection{Interface pressure calculation}

We consider now the assumptions of Eqs. (8) and (9) and no-flow boundary conditions imposed at the periphery of the computational domain. Therefore, using Eq. (24) in the continuous flux equation, Eq. (9), yields,

$$
\begin{aligned}
& L_{A}^{E} \lambda_{t}^{E^{n}} K^{E} P^{E^{n+1}}+L_{A}^{E^{\prime}} \lambda_{t}^{E^{\prime n}} K^{E^{\prime}} P^{E^{\prime n+1}} \\
& \quad=\lambda_{t}^{E^{n}} K^{E} \sum_{A^{\prime} \subset \partial E} B_{A, A^{\prime}}^{E^{-1}} P_{A^{\prime}}^{n+1}+\lambda_{t}^{E^{\prime n}} K^{E^{\prime}} \sum_{A^{\prime} \subset \partial E^{\prime}} B_{A, A^{\prime}}^{E^{\prime}-1} P_{A^{\prime}}^{n+1}
\end{aligned}
$$

To eliminate $P^{E^{n+1}}$ from Eqs. (26) and (28) and obtain the equation for the interface pressure unknowns, $I P_{A}^{n+1}$, we multiply Eq. (26) by $\frac{\beta^{E}}{L^{E} \lambda_{t}^{E^{n}} K^{E}}$,

$$
P^{E^{n+1}}=\left(1-\beta^{E}\right) P^{E^{n}}+\beta^{E} \sum_{A \subset \partial E} \frac{L_{A}^{E}}{L^{E}} I P_{A}^{n+1}+\frac{\beta^{E}}{L^{E} \lambda_{t}^{E^{n}} K^{E}} F_{t}^{E^{n+1}}
$$

where

$$
\begin{aligned}
\beta^{E} & =\frac{\gamma^{E}}{1+\gamma^{E}} \\
\gamma^{E} & =L^{E} \frac{\lambda_{t}^{E^{n}} K^{E} \Delta t}{V_{p}^{E^{n} C_{r}^{E}}}
\end{aligned}
$$

Then, we multiply by $L_{A}^{E} \lambda_{t}^{E^{n}} K^{E}$,

$$
L_{A}^{E} \lambda_{t}^{E^{n}} K^{E} P^{E^{n+1}}=L_{A}^{E} \lambda_{t}^{E^{n}} K^{E}\left(1-\beta^{E}\right) P^{E^{n}}+\lambda_{t}^{E^{n}} K^{E} \sum_{A^{\prime} \subset \partial E} \sigma_{A, A^{\prime}}^{E} I P_{A^{\prime}}^{n+1}+\frac{\beta^{E} L_{A}^{E}}{L^{E}} F_{t}^{E^{n+1}}
$$

where

$$
\sigma_{A, A^{\prime}}^{E}=\beta^{E} \frac{L_{A}^{E} L_{A^{\prime}}^{E}}{L_{E}} \quad \forall A, A^{\prime} \subset \partial E
$$

We put Eq. (32) into Eq. (28) to obtain the equations for the interface pressure unknowns $I P_{A}^{n+1}$,

$$
\begin{aligned}
& \sum_{E \supset A} L_{A}^{E} \lambda_{t}^{E^{n}} K^{E}\left(1-\beta^{E}\right) P^{E^{n}}+\sum_{E \supset A} \lambda_{t}^{E^{n}} K^{E} \sum_{A^{\prime} \subset \partial E} \sigma_{A, A^{\prime}}^{E} I P_{A^{\prime}}^{n+1}+\sum_{E \supset A} \frac{\beta^{E} L_{A}^{E}}{L^{E}} F_{t}^{E^{n+1}} \\
& \quad=\sum_{E \supset A} \lambda_{t}^{E^{n}} K^{E} \sum_{A^{\prime} \subset \partial E} B_{A, A^{\prime}}^{E^{-1}} I P_{A^{\prime}}^{n+1}
\end{aligned}
$$

Finally, we rearrange Eq. (34) into the matrix form to obtain the final equation to solve interface pressure unknowns,

$$
(\mathbf{M}-\mathbf{N}) \mathbf{I P}^{n+1}=\mathbf{F}
$$

where 


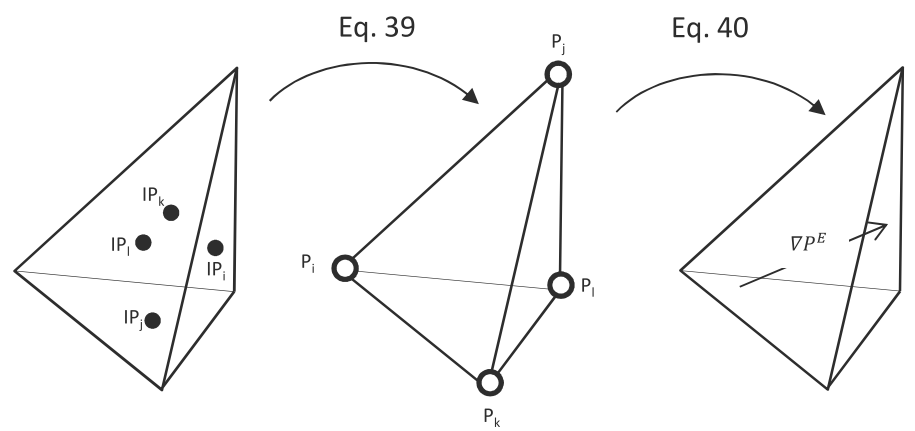

Fig. 4. Steps in calculating tetrahedron element pressure derivatives.

$$
\begin{aligned}
& M_{A, A^{\prime}}=\sum_{E \supset A \text { and } A^{\prime}} \lambda_{t}^{E^{n}} K^{E} B_{A, A^{\prime}}^{E^{-1}} \\
& N_{A, A^{\prime}}=\sum_{E \supset A \text { and } A^{\prime}} \lambda_{t}^{E^{n}} K^{E} \sigma_{A, A^{\prime}}^{E} \\
& F_{A}=\sum_{E \supset A}\left(L_{A}^{E} \lambda_{t}^{E^{n}} K^{E}\left(1-\beta^{E}\right) P^{E^{n}}+\frac{\beta^{E} L_{A}^{E}}{L^{E}} F_{t}^{E^{n+1}}\right)
\end{aligned}
$$

Once the interface pressures are calculated, the next step is to calculate the pressure derivative in each element which is discussed in the next section.

\subsection{Element pressure derivative}

Once the interface pressure is calculated, we can approximate the node pressures in each element assuming the interface pressure is at the centroid of each interface and the node pressures are discontinuous between the elements,

$$
\left[\begin{array}{c}
P_{i}^{E} \\
P_{j}^{E} \\
P_{k}^{E} \\
P_{l}^{E}
\end{array}\right]=\left[\begin{array}{cccc}
-2 & 1 & 1 & 1 \\
1 & -2 & 1 & 1 \\
1 & 1 & -2 & 1 \\
1 & 1 & 1 & -2
\end{array}\right]\left[\begin{array}{c}
I P_{i}^{E} \\
I P_{j}^{E} \\
I P_{k}^{E} \\
I P_{l}^{E}
\end{array}\right]
$$

where $P_{i}^{E}$ is node $i$ pressure of element $E$. Then, using first-order Courant basis function derivatives [28] we calculate the pressure drop vector necessary to calculate the fluid velocity vector, see Fig. 4:

$$
\nabla P^{E}=\left[\begin{array}{cccc}
\frac{\partial N_{i}^{E}}{\partial x} & \frac{\partial N_{j}^{E}}{\partial x} & \frac{\partial N_{k}^{E}}{\partial x} & \frac{\partial N_{l}^{E}}{\partial x} \\
\frac{\partial N_{i}^{E}}{\partial y} & \frac{\partial N_{j}^{E}}{\partial y} & \frac{\partial N_{k}^{E}}{\partial y} & \frac{\partial N_{l}^{E}}{\partial y} \\
\frac{\partial N_{i}^{E}}{\partial z} & \frac{\partial N_{j}^{E}}{\partial z} & \frac{\partial N_{k}^{E}}{\partial z} & \frac{\partial N_{l}^{E}}{\partial z}
\end{array}\right]\left[\begin{array}{c}
P_{i}^{E} \\
P_{j}^{E} \\
P_{k}^{E} \\
P_{l}^{E}
\end{array}\right]
$$

where

$$
\begin{aligned}
\frac{N_{i}^{E}}{\partial x} & =\frac{-[(\mathbf{K}-\mathbf{J}) \times(\mathbf{L}-\mathbf{J})]_{x}}{(\mathbf{J}-\mathbf{I}) \cdot((\mathbf{K}-\mathbf{J}) \times(\mathbf{L}-\mathbf{J}))} \\
\frac{N_{j}^{E}}{\partial x} & =\frac{-[(\mathbf{L}-\mathbf{K}) \times(\mathbf{I}-\mathbf{K})]_{x}}{(\mathbf{K}-\mathbf{J}) \cdot((\mathbf{L}-\mathbf{K}) \times(\mathbf{I}-\mathbf{K}))} \\
\frac{N_{k}^{E}}{\partial x} & =\frac{-[(\mathbf{I}-\mathbf{L}) \times(\mathbf{J}-\mathbf{L})]_{x}}{(\mathbf{L}-\mathbf{K}) \cdot((\mathbf{I}-\mathbf{L}) \times(\mathbf{J}-\mathbf{L}))} \\
\frac{N_{l}^{E}}{\partial x} & =\frac{-[(\mathbf{J}-\mathbf{I}) \times(\mathbf{K}-\mathbf{I})]_{x}}{(\mathbf{I}-\mathbf{L}) \cdot((\mathbf{J}-\mathbf{I}) \times(\mathbf{K}-\mathbf{I}))}
\end{aligned}
$$

where $\frac{\partial N_{i}^{E}}{\partial x}$ is the first-order Courant basis function derivative in the $x$-direction for node $i$ in element $E$, $\mathbf{I}$ is the coordinates of node $i$ for tetrahedron element $E$, and $[\mathbf{r}]_{x}$ indicates the $x$-component of the vector $\mathbf{r}$. Similar expressions are for $y$ and $z$ direction derivatives. 

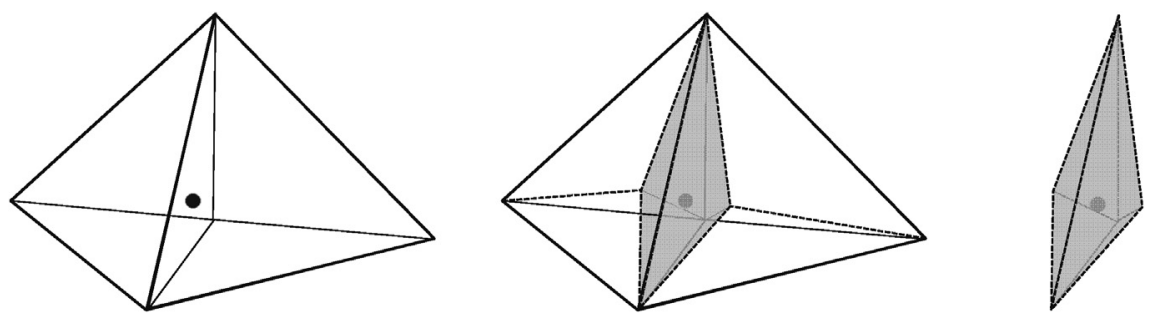

Fig. 5. Two tetrahedron elements sharing an interface (left), an interface control volume (grey colour) composed of two tetrahedron-shaped volumes connected at their bases, see Fig. 6, on the shared interface with their apex at the barycentre of host elements (centre), one interface control volume (right).
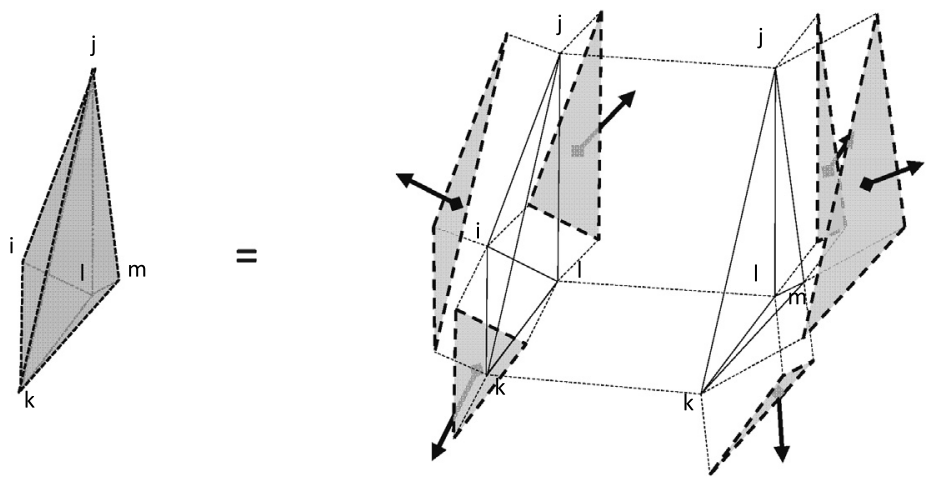

Fig. 6. Area normal vectors of the faces of an interface control volume for the ICVFE method.

\subsection{Construction of an interface control volume mesh}

The control volume mesh is a secondary mesh imposed on the finite element mesh to assure continuous fluxes and mass conservation during fluid advection. In the ICVFE method, the control volumes are constructed around element interfaces, Fig. 5. An interface control volume between two tetrahedron elements is formed by two tetrahedron-shaped volumes connected at their bases on the shared interface with their apex at the barycentre of the host elements, Figs. 5 and 6 . It is worth mentioning that the fluxes on the elements interfaces are continuous, Eq. (9). Here we are calculating the advection on the interface control volumes not on the elements (the case for MHFE).

Once the interface-control volumes are constructed, the area normal vector (AN) of each control volume face is needed for flux calculation. Fig. 6 shows a typical interface-control volume. It has five vertexes, $(i, j, k, l, m)$, and six sectors (faces). The calculation of the AN of each sector with the vector pointing outwards is given below,

$$
\begin{aligned}
& \mathbf{A N}_{i j k}=\left\{\begin{array}{ll}
0.5(\mathbf{J}-\mathbf{I}) \times(\mathbf{K}-\mathbf{I}) & \text { if } T_{l i j k}>0 \\
-0.5(\mathbf{J}-\mathbf{I}) \times(\mathbf{K}-\mathbf{I}) & \text { if } T_{l i j k}<0
\end{array} \quad i, j, k, l\right. \text { all different } \\
& \mathbf{A N}_{j k m}=\left\{\begin{array}{ll}
0.5(\mathbf{K}-\mathbf{J}) \times(\mathbf{M}-\mathbf{J}) & \text { if } T_{l j k m}>0 \\
-0.5(\mathbf{K}-\mathbf{J}) \times(\mathbf{M}-\mathbf{J}) & \text { if } T_{l j k m}<0
\end{array} \quad j, k, m, l\right. \text { all different }
\end{aligned}
$$

where

$$
\begin{aligned}
& T_{l i j k}=(\mathbf{I}-\mathbf{L}) \cdot[(\mathbf{J}-\mathbf{I}) \times(\mathbf{K}-\mathbf{I})] \\
& T_{l j k m}=(\mathbf{J}-\mathbf{L}) \cdot[(\mathbf{K}-\mathbf{J}) \times(\mathbf{M}-\mathbf{J})]
\end{aligned}
$$

\subsection{Fluid advection}

To calculate the advection of fluid between the control volumes, we integrate the transport equation Eq. (1) over control volume $A$ and apply the divergence theorem and implicit Euler discretisation of time,

$$
S_{\alpha, A}^{n+1}=\frac{S_{\alpha, A}^{n} V_{p, A}^{n}+\Delta t\left[\sum_{j=1}^{s i} \mathbf{v}_{\alpha, j} \cdot \mathbf{A N}_{j}+F_{\alpha, A}^{n+1}\right]}{V_{p, A}^{n+1}}
$$

where $S_{\alpha, A}$ is phase saturation in control volume $A, \mathbf{A N}$ is the sector area normal, Eqs. (45) and (46), SI is the number of sectors in control volume $A$, and $j$ is the sector number, $\mathbf{v}_{\alpha, j}$ is the phase velocity in sector $j$ calculated using Eqs. (2) and (40). The phase mobility, Eq. (3), is calculated using the saturation of upstream direction. 


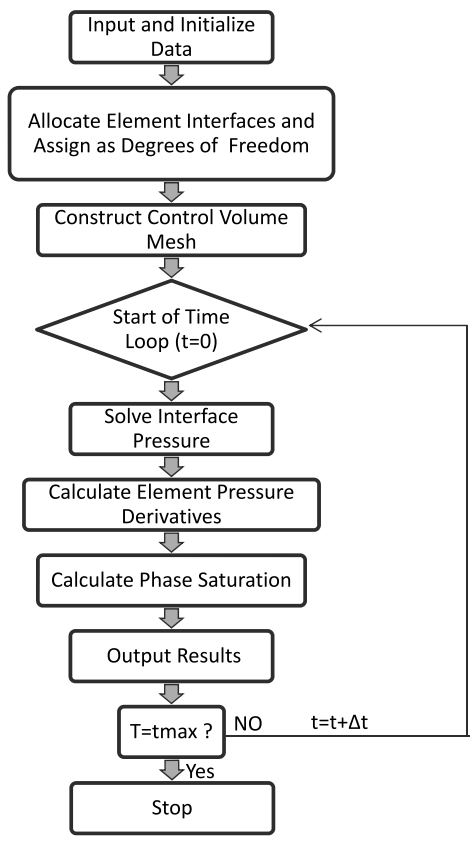

Fig. 7. Flow chart for the interface control volume finite element method.

\subsection{ICVFE implementation algorithm}

To conclude this section, we present the implementation algorithm chart of ICVFE, Fig. 7. We solve the pressure implicitly and saturation explicitly (IMPES). The fluid mobility needed for pressure equation is arithmetically averaged between the shared control volumes of each element. The degrees of freedom of the system are the number of interfaces in the mesh.

\section{Numerical tests}

To benchmark the performance of the ICVFE method, we present numerical tests with a broad range of material properties and boundary conditions. First, we benchmark its multi-phase convergence versus the Buckley-Leverett analytical solution. Second, we impose a zero permeability zone in the middle of a domain to test its reduction of artificial fluid smearing. Third, we test its fracture representation capabilities by imposing a fracture in the middle of a domain. The last test deals with a heterogeneous layer with large variations in the permeability field. In all the tests, we use threedimensional meshes made of tetrahedron finite elements created using the open source software GMSH [33]. The meshes are refined uniformly by changing the element length $(h)$ parameter in GMSH. The porosity is defined as 0.2 and we assume quadratic relative permeability curves for oil and water $\left(N_{o}=N_{w}=2\right)$ with $k_{r w}^{0}=0.7$. The water and oil viscosities are 0.4 and $2.5 \mathrm{mPa}$ s respectively. The rock compressibility equals $4.0 \times 10^{-10} \mathrm{~Pa}^{-1}$ and the medium is initially fully saturated with oil. Some of the simulation results are presented as a function of pore volume injected (PVI) and it is defined as,

$$
P V I=\frac{1}{V_{p}} \int_{0}^{t} F_{t} d t
$$

where $V_{p}$ is the total pore volume of the system.

We also compare the ICVFE method to the NCVFE method using meshes of similar degrees of freedom. The degrees of freedom for NCVFE are the nodes and the degrees of freedom for ICVFE are the interfaces. The deviation from reference solution, error, is calculated for each test.

\subsection{Test I: Buckley-Leverett case}

A multi-phase problem reduces to the Buckley-Leverett problem when gravity and capillarity are negligible [34,35]. We compare the ICVFE method to the one-dimensional analytical solution of the Buckley-Leverett problem using five meshes with homogeneous material properties. The domain is rectangular tube with dimensions of $1 \mathrm{~m} \times$ Element Length $\times$ Element Length. The meshes are three-dimensional with uniform and isotropic tetrahedron finite elements. The properties of the meshes are shown in Table 1. Fig. 8 compares the numerical solution of the meshes versus the analytical solution. 
Table 1

Error in Buckley-Leverett problem for ICVFE and NCVFE methods with various meshes.

\begin{tabular}{|c|c|c|c|c|c|}
\hline $\begin{array}{l}\text { Element length }(h) \\
{[\mathrm{m}]}\end{array}$ & Elements & Interfaces & Nodes & $L_{2}($ ICVFE) & $L_{2}(\mathrm{NCVFE})$ \\
\hline 0.1 & 62 & 164 & 42 & 0.116 & 0.141 \\
\hline 0.05 & 122 & 324 & 82 & 0.082 & 0.122 \\
\hline 0.0125 & 482 & 1284 & 322 & 0.035 & 0.067 \\
\hline 0.0025 & 2410 & 6420 & 1602 & 0.016 & 0.028 \\
\hline 0.001 & 6002 & 16,004 & 4002 & 0.009 & 0.017 \\
\hline
\end{tabular}

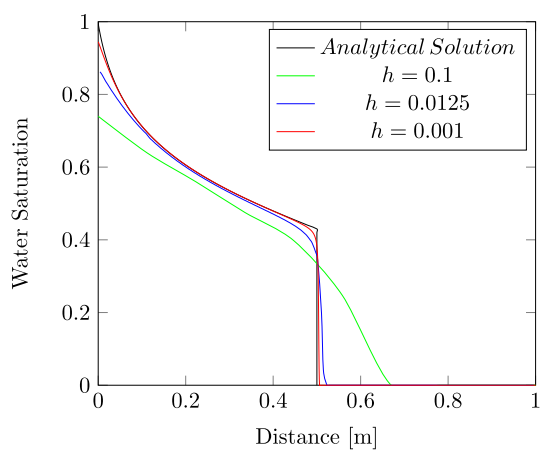

Fig. 8. Comparison of the analytical solution of the Buckley-Leverett problem at distance $0.5 \mathrm{~m}$ from the left-hand boundary to the numerical solution of the ICVFE method using three meshes, see Table 1. Water is injected at the left and displaces oil in a homogeneous porous medium.

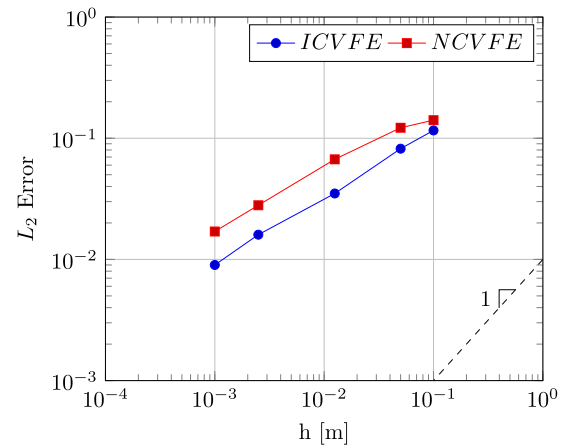

Fig. 9. Convergence of the $L_{2}$ error of water saturation as a function of the mesh element length for the numerical solutions of the Buckley-Leverett problem using the ICVFE and NCVFE methods, see Table 1.

As the mesh is refined the numerical solution of ICVFE method converges to the analytical solution. Fig. 9 shows the convergence of the error $L_{2}$ for the saturation profiles shown in Fig. 8:

$$
L_{2}=\left[\frac{1}{N}\left(\sum_{j=1}^{N}\left|S_{w, j}-S_{w}^{r e f}\left(x_{j}\right)\right|^{2}\right)\right]^{0.5}
$$

where $N$ is the degrees of freedom in the flow direction. We also calculate the error produced by the NCVFE method for this test. The ICVFE method models more accurate water saturation profiles for the same element length (Fig. 9 and Table 1). We can define a convergence rate $p$,

$$
L_{2} \sim h^{p}
$$

where $h$ is element length. Using Eq. (52), we calculate convergence rates of approximately 0.55 for ICVFE and 0.45 for NCVFE methods. These relatively modest convergence rates are a result of the shock between the two fluids that dominates the overall error. Schmid et al. [7] and Hoteit and Firoozabadi [36] applied the Buckley-Leverett problem for various numerical methods. They observed low convergence rates for the Buckley-Leverett problem regardless of the numerical method used, as we see here, because of this sharp separation between the two fluids. 


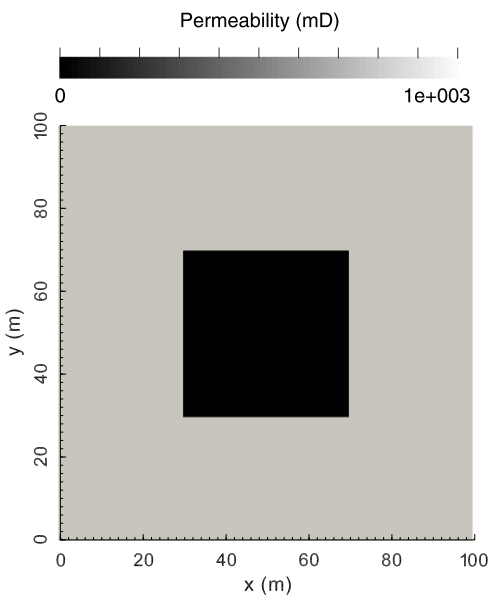

(a) Top view

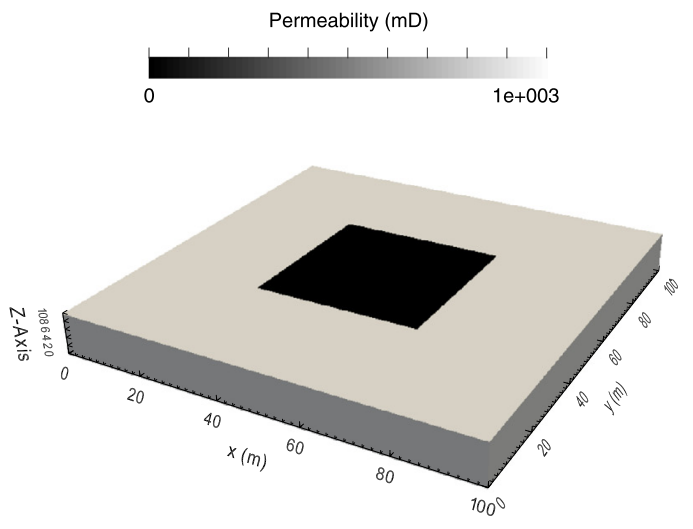

(b) Side view

Fig. 10. Domain of Test II; there is a no-flow (zero permeability) region in the centre.

Table 2

Error in water saturation in the zero permeability zone of Test II for ICVFE and NCVFE methods for various meshes.

\begin{tabular}{|c|c|c|c|c|c|c|c|}
\hline \multirow{3}{*}{$\begin{array}{l}\text { Element } \\
\text { length }(h) \\
{[\mathrm{cm}]}\end{array}$} & \multirow[t]{3}{*}{ Elements } & \multirow[t]{3}{*}{ Interfaces } & \multirow[t]{3}{*}{ Nodes } & \multicolumn{4}{|l|}{$e\left(S_{w}\right)$} \\
\hline & & & & \multicolumn{2}{|c|}{0.3 PVI } & \multicolumn{2}{|c|}{0.6 PVI } \\
\hline & & & & ICVFE & NCVFE & ICVFE & NCVFE \\
\hline 30 & 237 & 498 & - & 0.016 & - & 0.038 & - \\
\hline 15 & 473 & 1122 & - & 0.014 & - & 0.034 & - \\
\hline 10 & 1503 & 3350 & 455 & 0.006 & 0.064 & 0.018 & 0.131 \\
\hline 6 & 4496 & 10,168 & 1350 & 0.004 & 0.049 & 0.013 & 0.116 \\
\hline 4 & 14,342 & 30,672 & 3471 & 0.002 & 0.029 & 0.009 & 0.057 \\
\hline 2.3 & 43,027 & - & 10,062 & - & 0.017 & - & 0.048 \\
\hline 1.5 & 172,079 & - & 33,599 & - & 0.009 & - & 0.032 \\
\hline
\end{tabular}

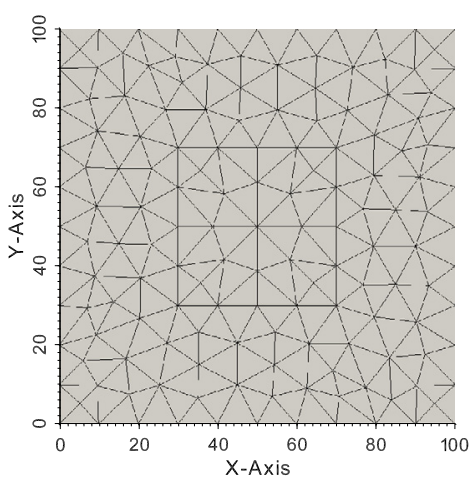

Fig. 11. Finite element mesh for the domain of Test II using element length of $10 \mathrm{~cm}$ (see Table 2).

\subsection{Test II: zero-permeability zone}

In this test, we introduce a zero permeability zone in the middle of a domain to test the smearing reduction in the ICVFE method and compare it to NCVFE. The domain is square with dimensions of $100 \mathrm{~cm} \times 100 \mathrm{~cm}$ and a depth of $10 \mathrm{~cm}$ with a central zero permeability zone $30 \mathrm{~cm}$ away from the side boundaries, Fig. 10. Water is injected at a constant rate along the left-hand boundary and we use different mesh resolutions of the domain. Table 2 shows the properties of the meshes. An example mesh is shown in Fig. 11. We compute the error in water saturation invading the zero permeability zone using

$$
e\left(S_{w}\right)=\frac{1}{V_{z}} \int\left|S_{w}-S_{w}^{r e f}\right| d v
$$



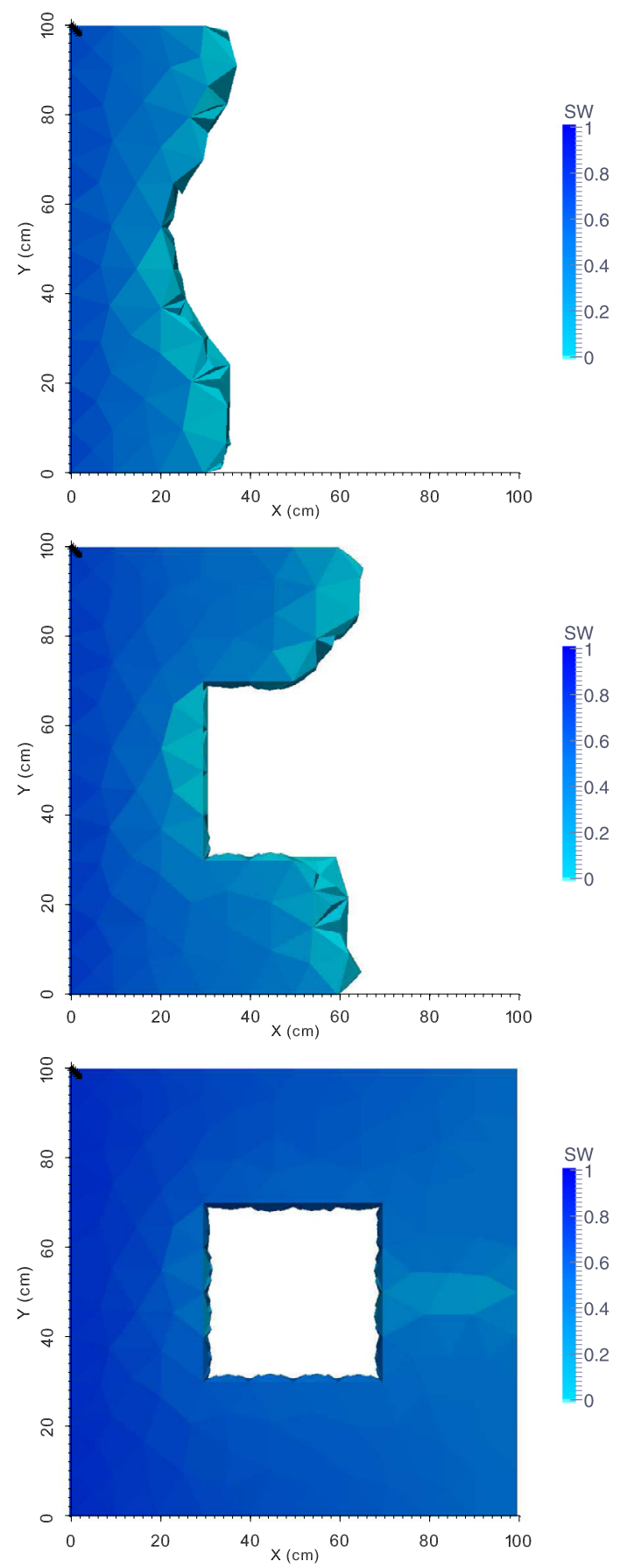
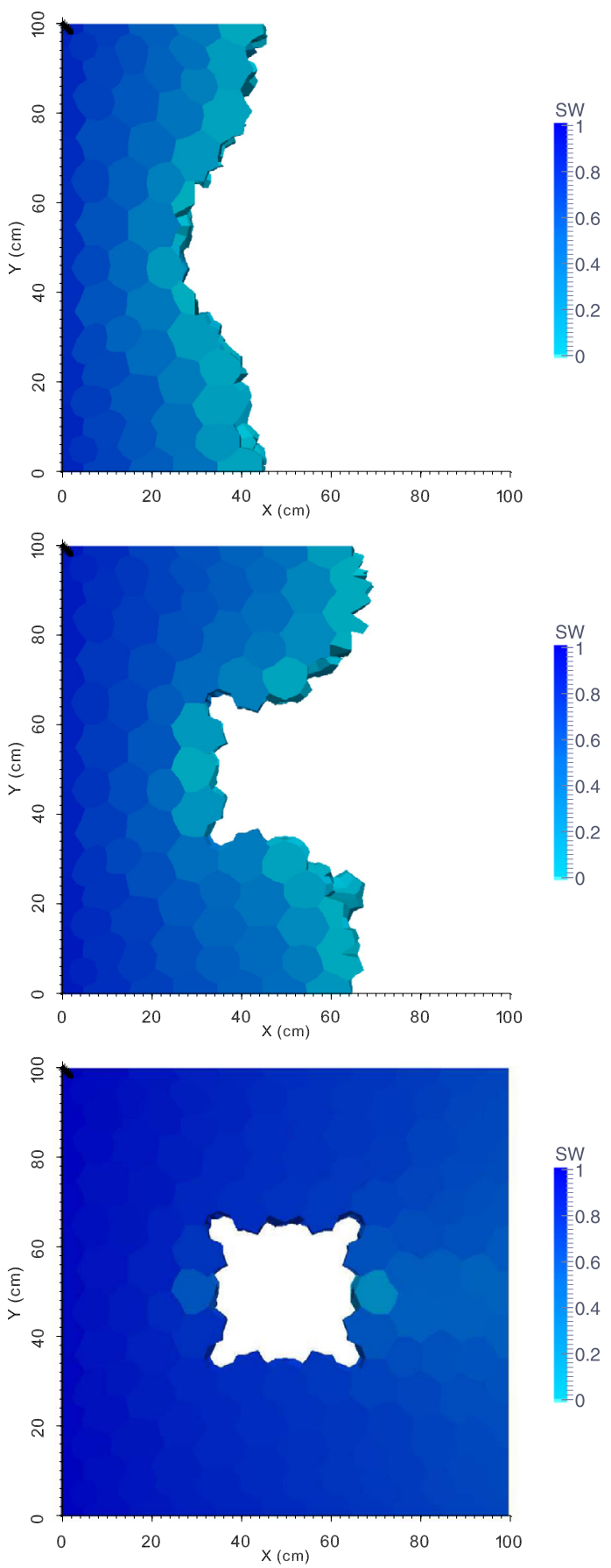

Fig. 12. Water saturation field modelled using ICVFE (left) and NCVFE (right) for Test II using mesh of element size $10 \mathrm{~cm}$ (see Table 2 and Fig. 11 ) at pore volumes injected of: 0.1 (top), 0.2 (centre), 0.6 (bottom).

where $V_{z}$ is the total pore volume of the zero permeability zone, $S_{w}^{r e f}$ is the reference solution for water saturation in the zone which equals the initial water saturation.

Fig. 12 shows the water saturation field employing the ICVFE and NCVFE methods at different values of pore volume injected for a mesh of element length $10 \mathrm{~cm}$, Fig. 11. The water modelled by ICVFE flows around the zero-permeability zone with minimal invasion. On the other hand, the water movement modelled by NCVFE heavily invades the side boundaries of the zero-permeability zone. The reduction of water saturation smearing in the zero permeability zone by ICVFE is good compared to NCVFE. Fig. 13 and Table 2 quantify this effect by showing the error in water saturation in the zero permeability 


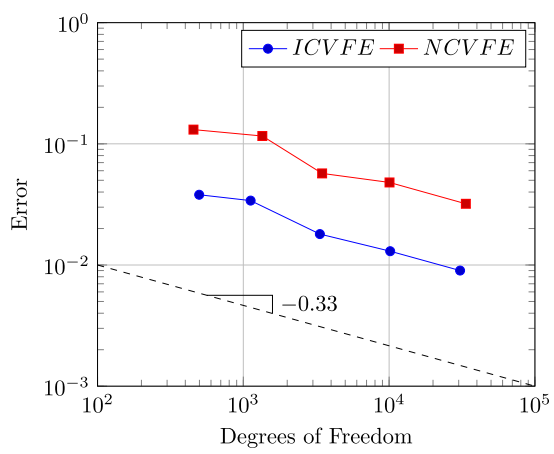

Fig. 13. Comparison of error in water saturation inside the impermeable zone for Test II between ICVFE and NCVFE methods at 0.6 pore volumes injected as a function of the mesh degrees of freedom. The ICVFE method produces less error than NCVFE for roughly the same number of degrees of freedom.

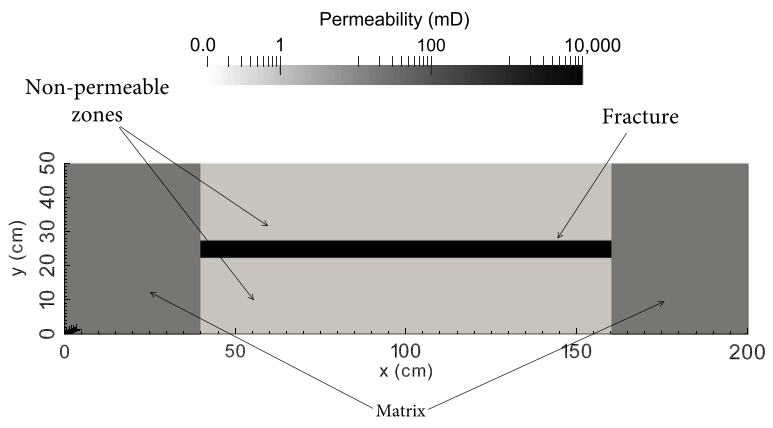

(a) Top view

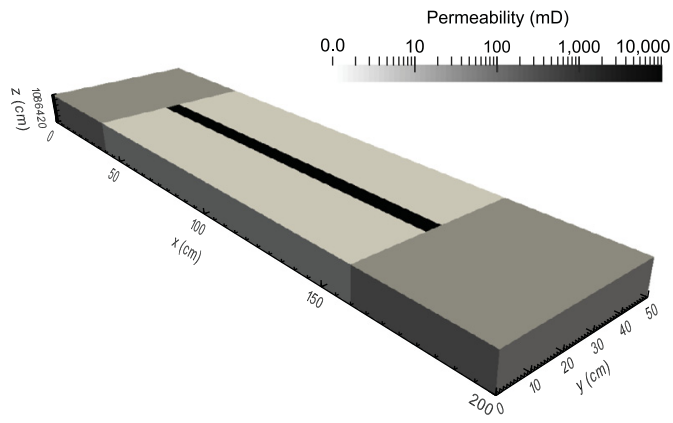

(b) Side view

Fig. 14. Domain of Test III; there is a fracture region in the centre (black) with non-permeable levees.

zone calculated by Eq. (53). Similar to the Buckley-Leverett problem, we measure the convergence rate. Here the domain is composed of unstructured elements; therefore we can define the convergence in terms of the degrees of freedom [37],

$$
e \sim N_{D F}^{p / d}
$$

where $N_{D F}$ is the number of degrees of freedom, $p$ is the order of convergence and $d$ is the spatial dimension of the problem.

Using Eq. (54), we find $p \approx 1$ for both methods (where $d=3$ ). This is an improved, linear, rate of convergence compared to the Buckley-Leverett case, since it is controlled by the level of numerical smearing into the zero-permeability zone rather than by the resolution of the sharp discontinuities between two fluids.

In this test, we find the ICVFE method produces less error than NCVFE for roughly the same number of degrees of freedom. This superior modelling of sealed zones by ICVFE compared to NCVFE is because ICVFE constructs the control volumes around the interfaces of element where, at most, two elements are shared. This limits the unphysical flow and produces more accurate results. On the other hand, the NCVFE method constructs the control volumes around the vertices of elements where several elements are shared. This promotes unphysical fluid smearing between elements with different material properties as we have shown in this case.

\subsection{Test III: one fracture case}

In this case, we introduce a narrow conductive zone (representing a fracture) in the middle of a domain to test the conductive capability of the ICVFE. The fracture has dimensions of $120 \times 5 \times 10 \mathrm{~cm}$, see Fig. 14 . Water is injected at a constant rate along the left-hand boundary. We mesh the domain using tetrahedron elements and we use different mesh resolutions. Table 3 shows the properties of the meshes. An example mesh is shown in Fig. 15.

To assess the extent of the unphysical flow produced by the ICVFE method better, we make the sides of the fracture zone (levees) non-permeable. This produces as reference solution that models the water saturation flowing from the matrix through the fracture zone to the right hand boundary without invading the levees. As indicated in Fig. 14, the fracture has a permeability of $10,000 \mathrm{mD}$ while the matrix at both ends of the domain has a permeability of $10 \mathrm{mD}$. Fig. 16 shows the water saturation employing the ICVFE and NCVFE methods at three different values of pore volumes injected for a mesh with an element length of $7 \mathrm{~cm}$ (Fig. 15). ICVFE represents the fracture zone with less unphysical smearing into the levees 
Table 3

Error in water saturation in levees zone of Test III for ICVFE and NCVFE methods with various meshes.

\begin{tabular}{|c|c|c|c|c|c|c|c|}
\hline \multirow{3}{*}{$\begin{array}{l}\text { Element } \\
\text { length }(h) \\
{[\mathrm{cm}]}\end{array}$} & \multirow[t]{3}{*}{ Elements } & \multirow[t]{3}{*}{ Interfaces } & \multirow[t]{3}{*}{ Nodes } & \multicolumn{4}{|l|}{$e\left(S_{w}\right)$} \\
\hline & & & & \multicolumn{2}{|c|}{0.2 PVI } & \multicolumn{2}{|c|}{0.4 PVI } \\
\hline & & & & ICVFE & $\overline{\text { NCVFE }}$ & $\overline{\text { ICVFE }}$ & $\overline{\text { NCVFE }}$ \\
\hline 40 & 233 & 554 & - & 0.028 & - & 0.038 & - \\
\hline 15 & 1348 & 1534 & - & 0.023 & - & 0.031 & - \\
\hline 7 & 2187 & 5059 & 727 & 0.012 & 0.071 & 0.017 & 0.099 \\
\hline 5.5 & 4886 & 11,035 & 1454 & 0.008 & 0.058 & 0.013 & 0.081 \\
\hline 4 & 10,132 & 22,491 & 2806 & 0.007 & 0.051 & 0.011 & 0.073 \\
\hline 2.3 & 43,431 & - & 10,235 & - & 0.033 & - & 0.047 \\
\hline 1.6 & 124,527 & - & 26,588 & - & 0.023 & - & 0.033 \\
\hline
\end{tabular}

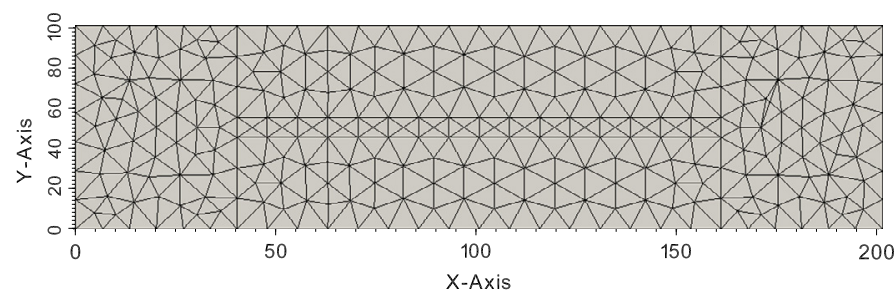

Fig. 15. Finite element mesh for the domain of Test III using an element length of $7 \mathrm{~cm}$ (see Table 3).
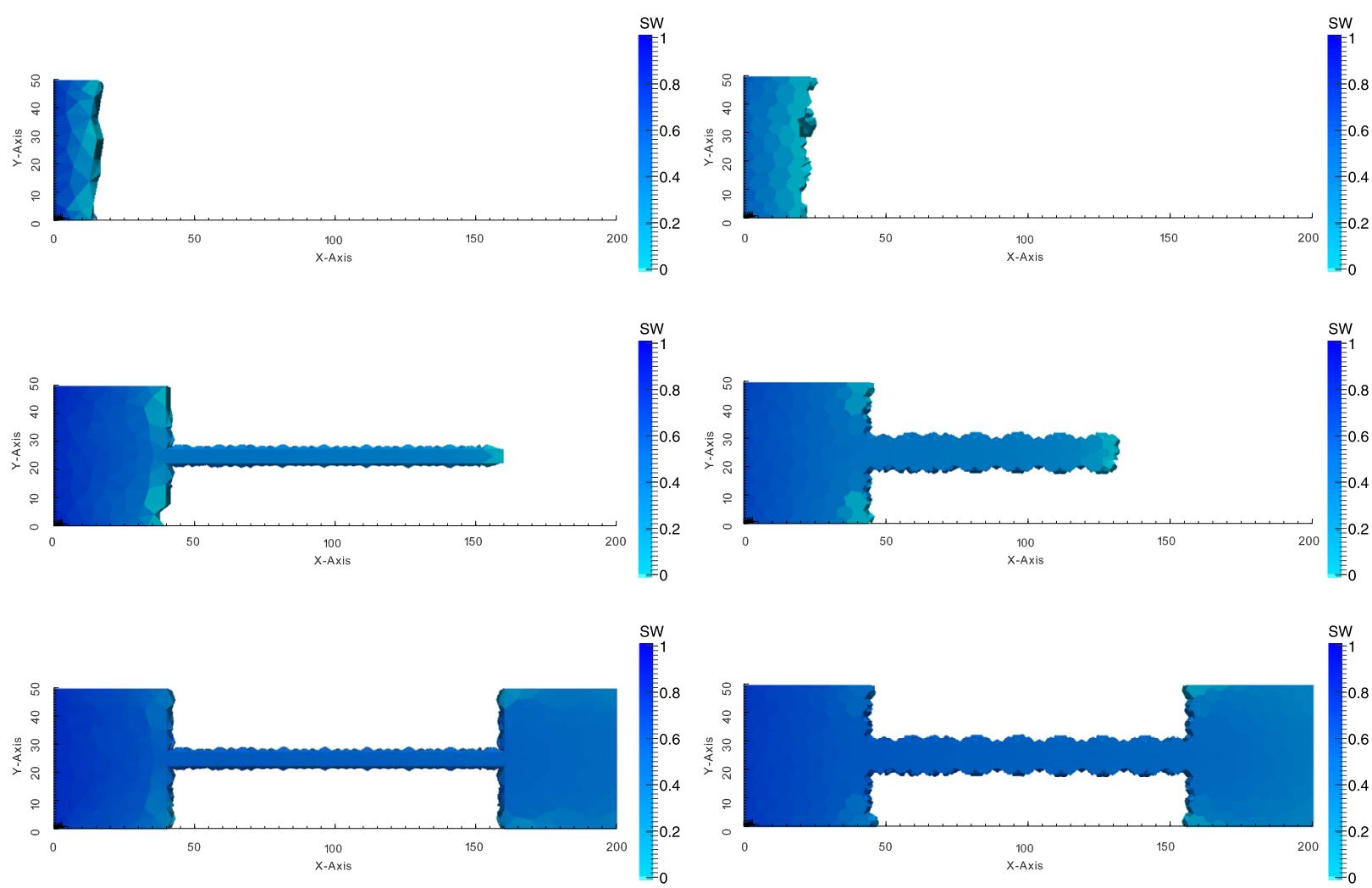

Fig. 16. Water saturation field modelled using ICVFE (left) and NCVFE (right) for Test III using mesh of element size $7 \mathrm{~cm}$ (see Table 3 and Fig. 15) at pore volumes injected of: 0.03 (top), 0.15 (centre), 0.4 (bottom).

region than NCVFE. The large artificial flow in NCVFE is due to the large control volumes constructed on the shared element nodes between both regions which delay the flow of water in the fracture region. To view this better, we plot a side view of this behaviour at 0.15 PVI in Fig. 17. We can see the large amount of artificial smearing compared to the ICVFE. The ICVFE 


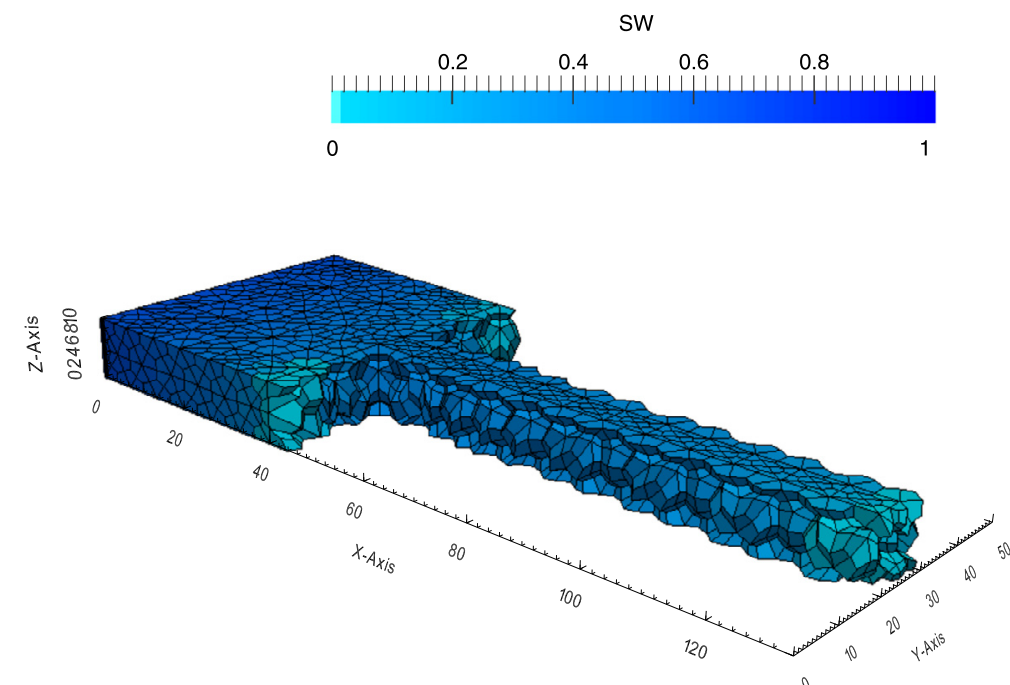

(a) NCVFE

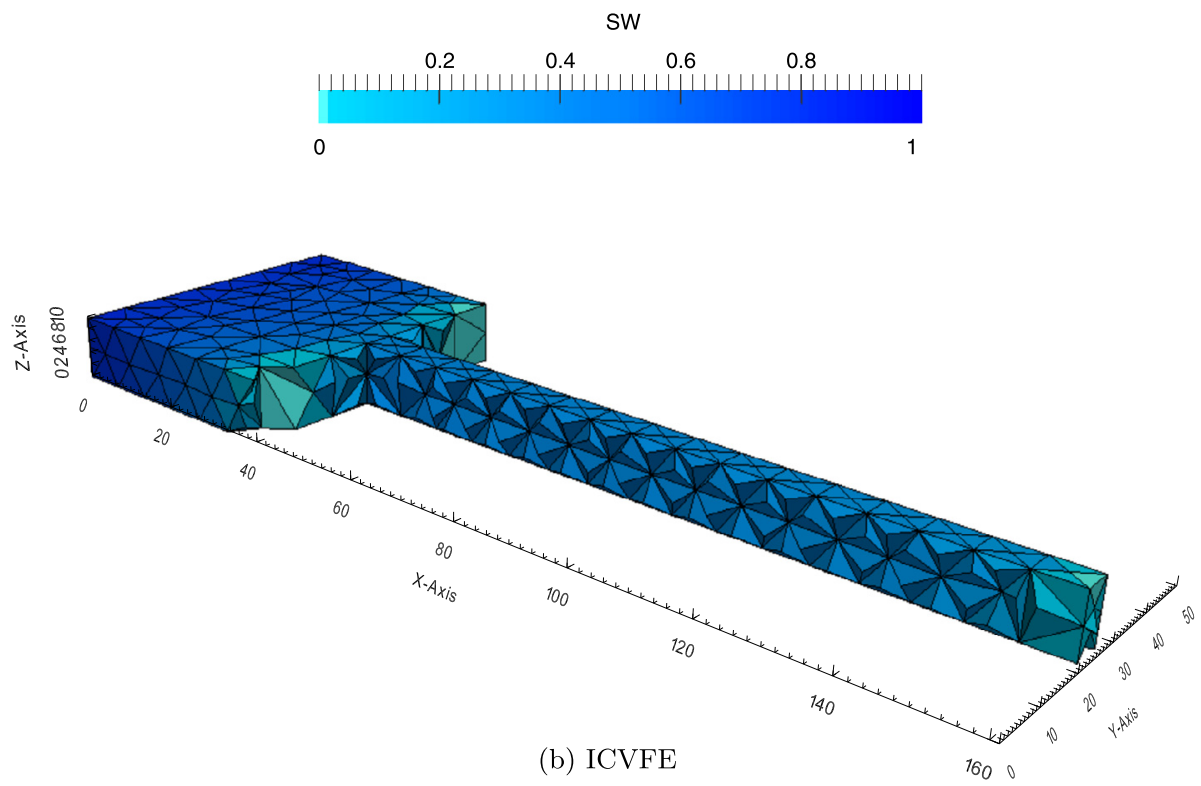

Fig. 17. A side view of the water saturation field modelled using NCVFE and ICVFE for Test III using mesh of element size $7 \mathrm{~cm}$ (see Table 3 and Fig. 14 ) at 0.15 pore volumes injected. The water saturation modelled by the ICVFE method is more confined and propagates faster in the fracture region with less unphysical flow into the levees than NCVFE.

method models a more confined fluid flow that promotes a quicker propagation it the fracture region with few unphysical flow thanks to the construction of control volumes on the interfaces of elements.

To quantify these effects, we calculate the error in water saturation in the levees regions using Eq. (53). Here the correct solution is zero invasion. Table 3 shows the error produced by both methods. The ICVFE produces less error than NCVFE for roughly the same degrees of freedom. Similar to the previous case, using Eq. (54), we find the convergence rate is of first-order for both methods where $d=3$, see Fig. 18 .

Fractures play a crucial role in hydrocarbon recovery. Their dimensions and aperture size (width) affect the kinetics and the final recovery of hydrocarbon reservoirs [38]. In this test, we saw how the fracture width was numerically extended (artificial smearing) by the NCVFE method prompting a delay of flow in the fracture region. This behaviour, if not modelled accurately, could lead to false predictions and inaccurate reservoir management. The ICVFE method models a more accurate behaviour of the flow with less ingress into the zero permeability domain. 


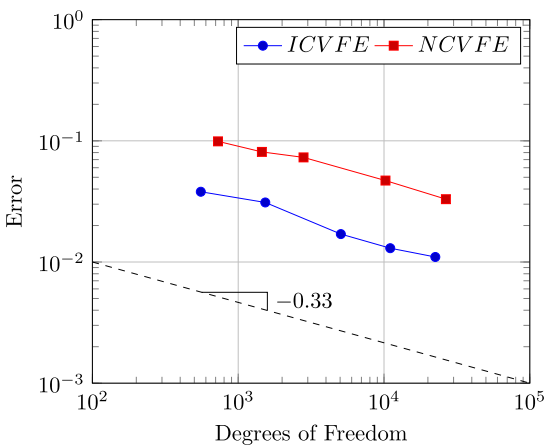

Fig. 18. Comparison of error in water saturation inside the levees zone for Test III between ICVFE and NCVFE methods at 0.4 pore volumes injected versus the meshes degrees of freedom. The ICVFE method produces less error than NCVFE.

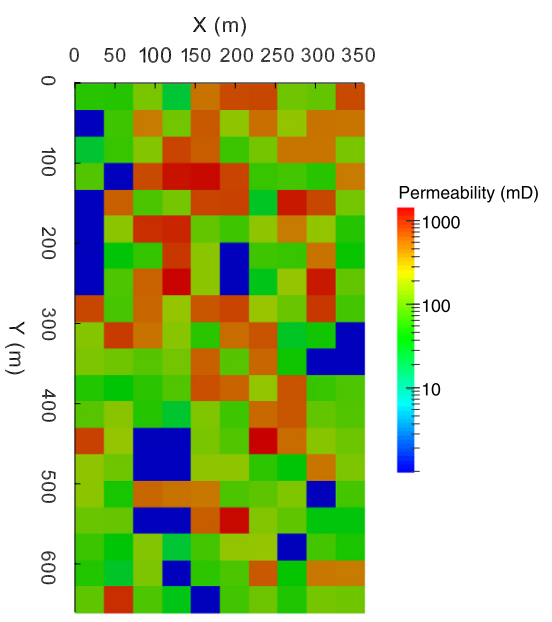

(a)

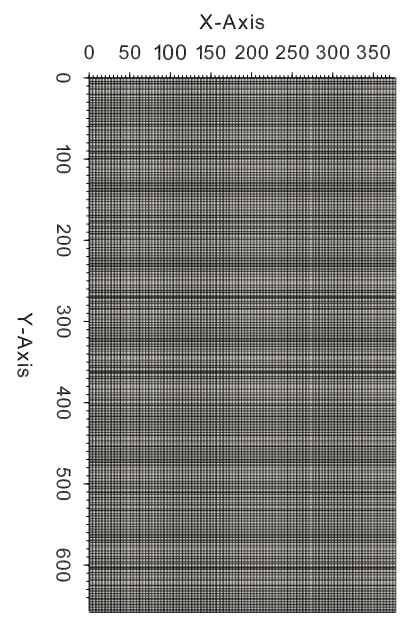

(b)

Fig. 19. (a) The permeability field of Test IV, (b) the fine scale mesh of the ECLIPSE reference solution $(100 \times 200 \times 3)$.

Table 4

The mesh properties of Test IV and the error of water saturation for ICVFE and NCVFE methods at two different pore volumes injected.

\begin{tabular}{|c|c|c|c|c|c|c|c|}
\hline \multirow{3}{*}{$\begin{array}{l}\text { Element } \\
\text { length }(h) \\
{[\mathrm{m}]}\end{array}$} & \multirow[t]{3}{*}{ Elements } & \multirow[t]{3}{*}{ Interfaces } & \multirow[t]{3}{*}{ Nodes } & \multicolumn{4}{|l|}{$\operatorname{re}\left(S_{w}\right)$} \\
\hline & & & & \multicolumn{2}{|c|}{$0.2 \mathrm{PVI}$} & \multicolumn{2}{|c|}{$0.4 \mathrm{PVI}$} \\
\hline & & & & $\overline{\text { ICVFE }}$ & $\overline{\text { NCVFE }}$ & ICVFE & $\overline{\text { NCVFE }}$ \\
\hline 36 & 1200 & 2860 & 462 & 0.228 & 0.445 & 0.215 & 0.403 \\
\hline 18 & 4800 & 11,320 & 1722 & 0.121 & 0.326 & 0.116 & 0.305 \\
\hline 12 & 10,800 & 25,380 & 3782 & 0.081 & 0.252 & 0.075 & 0.242 \\
\hline 9 & 19,200 & 45,040 & 6642 & 0.057 & 0.215 & 0.052 & 0.201 \\
\hline 6 & 43,200 & - & 14,762 & - & 0.162 & - & 0.149 \\
\hline 4 & 97,200 & - & 32,942 & - & 0.129 & - & 0.116 \\
\hline
\end{tabular}

\subsection{Test IV: heterogeneous layer}

In this case, we test the accuracy of the ICVFE method for modelling two-phase flow in heterogeneous systems. We introduce a layer of size $360 \times 660 \mathrm{~m}$ composed of $10 \times 20$ permeability regions ranging from 0 to $1436 \mathrm{mD}$, see Fig. 19 . We place one water injector and one oil producer at diagonally opposing corners (top-left and bottom-right), and water is injected and oil is produced at a same constant rate.

We mesh the domain using tetrahedron elements and we use different mesh resolutions. Table 4 shows the properties of the meshes. The reference solution for this test is computed numerically using commercial reservoir simulator ECLIPSE $[39,40]$ on a fine scale mesh composed of orthogonal grids with $10 \times 10 \times 3$ cell in each permeability region $(60,000$ degrees of freedom), see Fig. 19.

In Fig. 20, we compare the water saturation modelled by the ICVFE and NCVFE methods to the reference solution after 0.4 pore volumes of water have been injected in the layer. In this figure, we show the solution of two different meshes 
$\mathrm{X}$-Axis

050100150200250300350

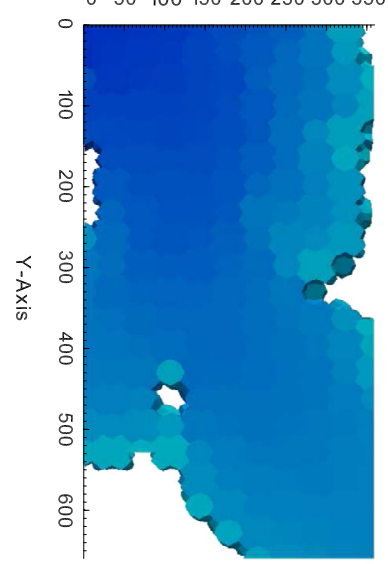

(a)

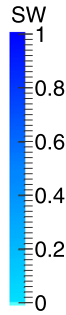

$\mathrm{X}$-Axis

50100150200250300350

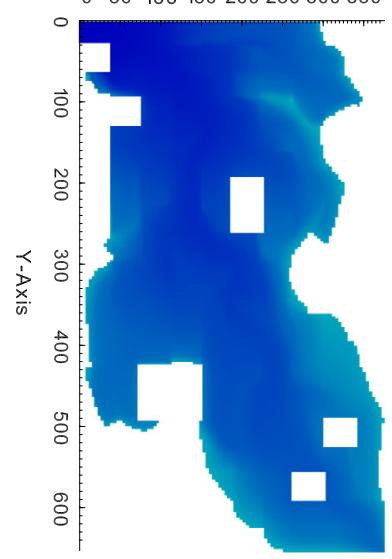

(c)

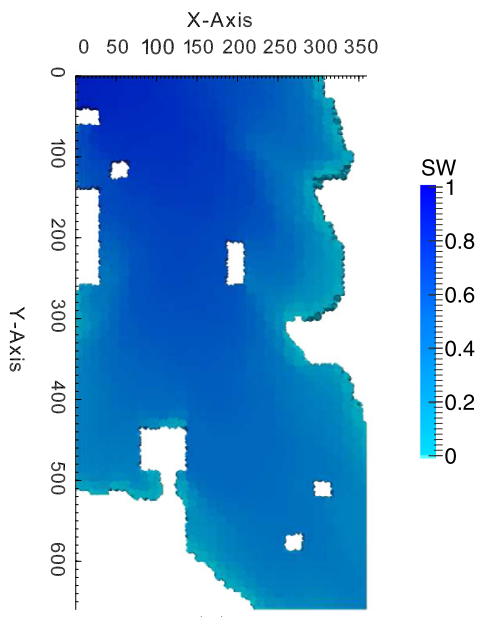

(b)

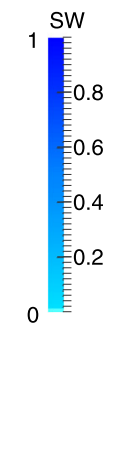

$\mathrm{X}$-Axis

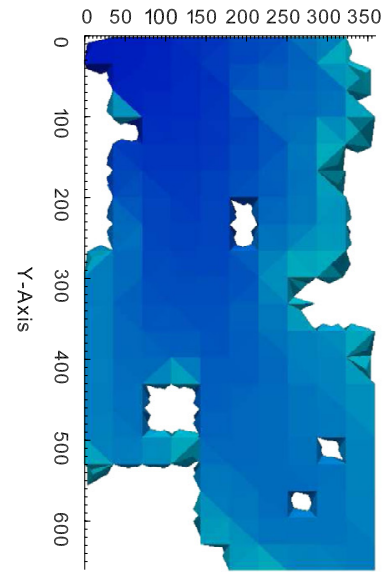

(d)

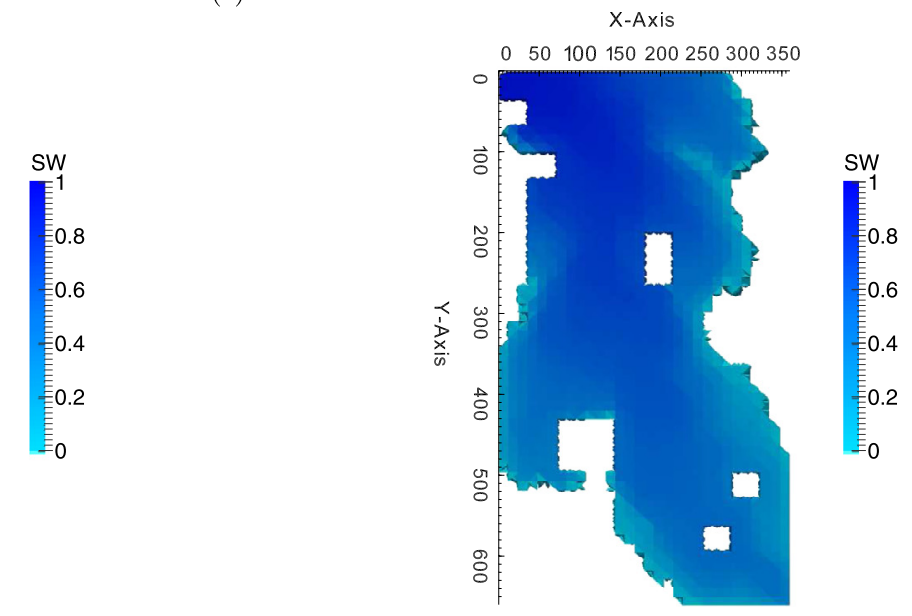

(e)

Fig. 20. The water saturation for Test IV at 0.4 pore volumes injected for: (a) NCVFE for mesh of element length 36 m, (b) NCVFE for mesh of element length $12 \mathrm{~m}$, (c) reference solution, (d) ICVFE for mesh of element length $36 \mathrm{~m}$, (e) ICVFE for mesh of element length $12 \mathrm{~m}$. 


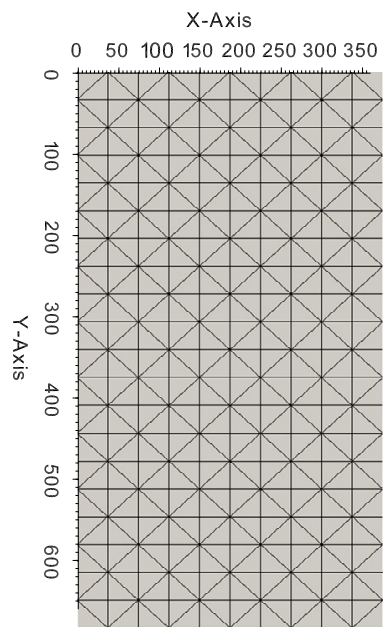

(a)

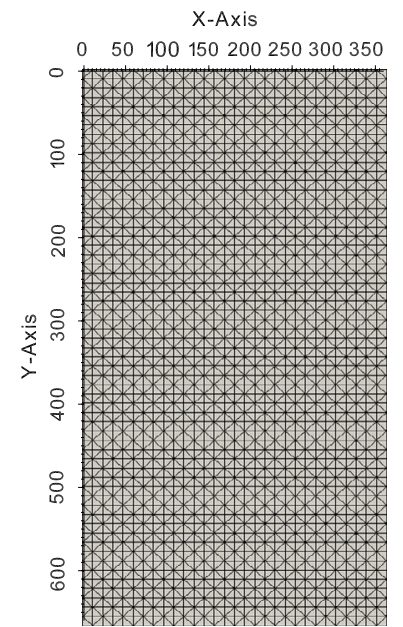

(b)

Fig. 21. The finite element mesh for the domain of Test IV using element length of: (a) $36 \mathrm{~m}$, (b) $12 \mathrm{~m}$ (see Table 4).

from Table 4, see Fig. 21. The ICVFE method produces an accurate representation of the water saturation in the heterogeneous layer compared to the reference solution, and the solution improves as the resolution of the mesh increases. On the other hand, the water modelled by the NCVFE method invades the low permeability regions (similar to Tests II and III) and produces a very poor model. The water dismisses the heterogeneity of the layer and sweeps most of the oil from the system. The solution improves as the mesh is refined. However, by looking at regions $(1,3)$ and $(1,4)$, we see the water invading these zero-permeability regions. This is physically inaccurate. The accurate representation is modelled by the ICVFE and reference solutions. The NCVFE method cannot avoid this, even with a higher mesh resolution as shown, since the control volumes are imposed around the edges of these two regions and will allow the water to flow between them.

To quantify these observations, we calculate the error in water saturation in each permeability region using Eq. (53), see Fig. 22. The NCVFE method produces large errors where neighbouring regions have large variations in their permeability, especially between regions $(1,3)$ and $(1,4)$. This error is less for the ICVFE method thanks to the control volumes being constructed around the interfaces of elements. For comparison and to measure the rate of convergence, we calculate the relative error in water saturation profiles for the whole layer [41] using

$$
\operatorname{re}\left(S_{w}\right)=\frac{\int\left|S_{w}-S_{w}^{r e f}\right| d v}{\int\left|S_{w}^{r e f}-S_{w_{i}}^{r e f}\right| d v}
$$

Fig. 23 shows this error for the ICVFE and NCVFE methods versus the degrees of freedom for the meshes listed in Table 4. Again, the ICVFE method produces less error than NCVFE. And using Eq. (35), we find the convergence rate $p$ is of first-order for ICVFE and equals 0.6 for NCVFE where $d=2$. This low convergence rate for the NCVFE method is because of the difficulty of this problem and the heavy artificial smearing between the heterogeneous permeability regions. The main issue with the NCVFE method is that the fluid saturation is computed on the vertices of elements and the material properties are defined on the elements. This particular two-mesh approach computes an up-scaled fluid saturation and is bound to produce unphysical flow in heterogeneous media, as we have seen in this test. Nick and Matthai [9] and Bazr-Afkan and Matthai [12] discuss this problem, and they developed novel numerical methods to improve the resolution of the fluid saturation. Essentially, this issue is what inspired us to develop the ICVFE method.

This test and the previous tests show the ICVFE method produces satisfactory and more physically accurate results than NCVFE for modelling multi-phase fluid flow in highly heterogeneous and fractured reservoirs. The ICVFE method models a down-scale (high resolution) representation of the water saturation since the number of interfaces is larger than the number of elements where the heterogeneity is defined. On the other hand, the performance of the NCVFE method is disappointing. It requires very high mesh resolutions to produce reasonable results but not necessarily physically accurate.

\section{Summary and conclusions}

We have presented a new finite element method called the interface control volume finite element (ICVFE) method. It improves modelling of multi-phase flow in highly heterogeneous and naturally fractured reservoirs. The associated ICVFE solution method approximates the pressure at the interfaces of elements and computes the advection of fluid on control 


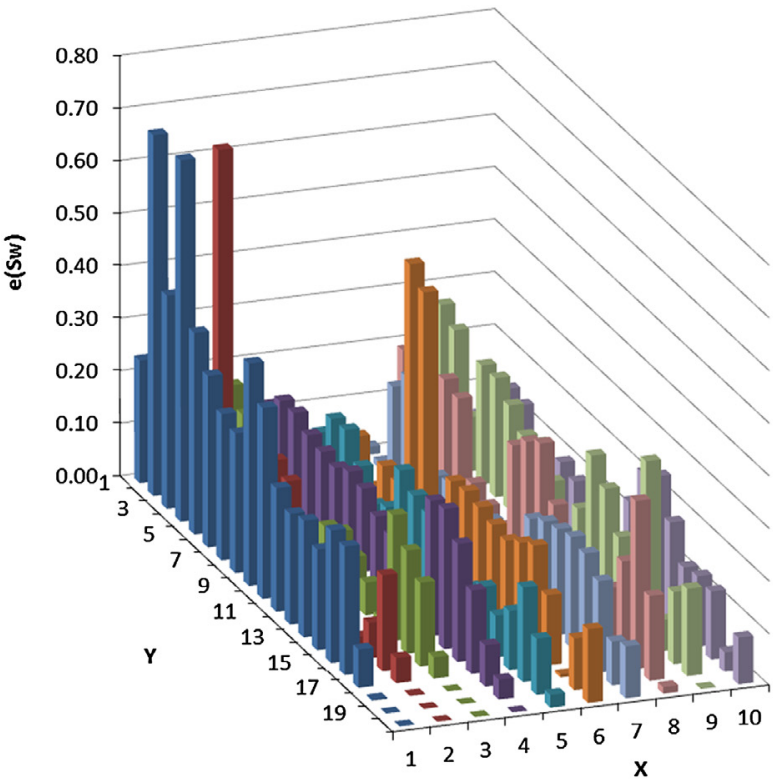

(a)

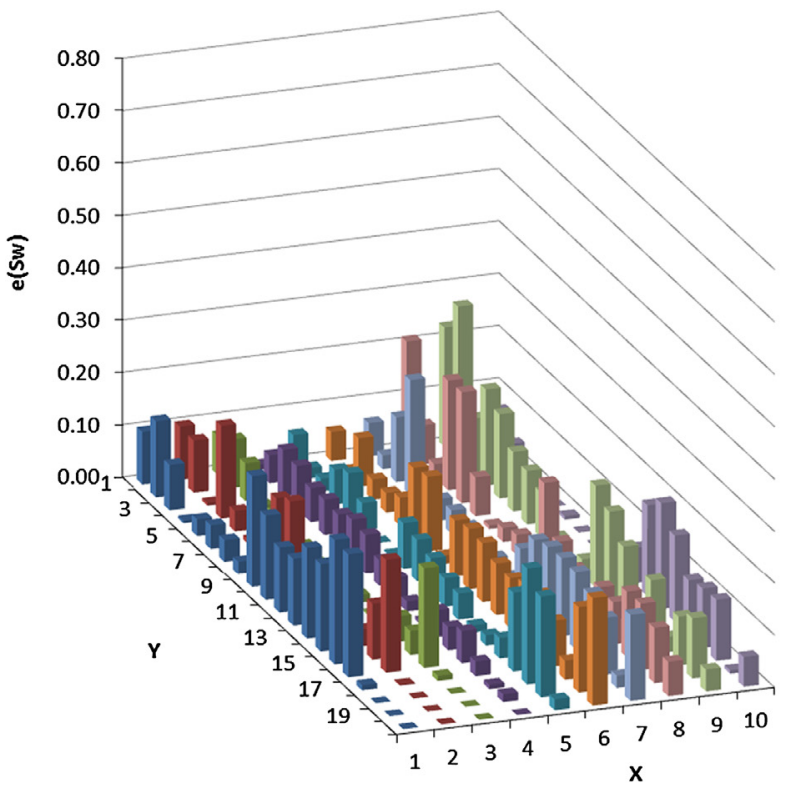

(c)

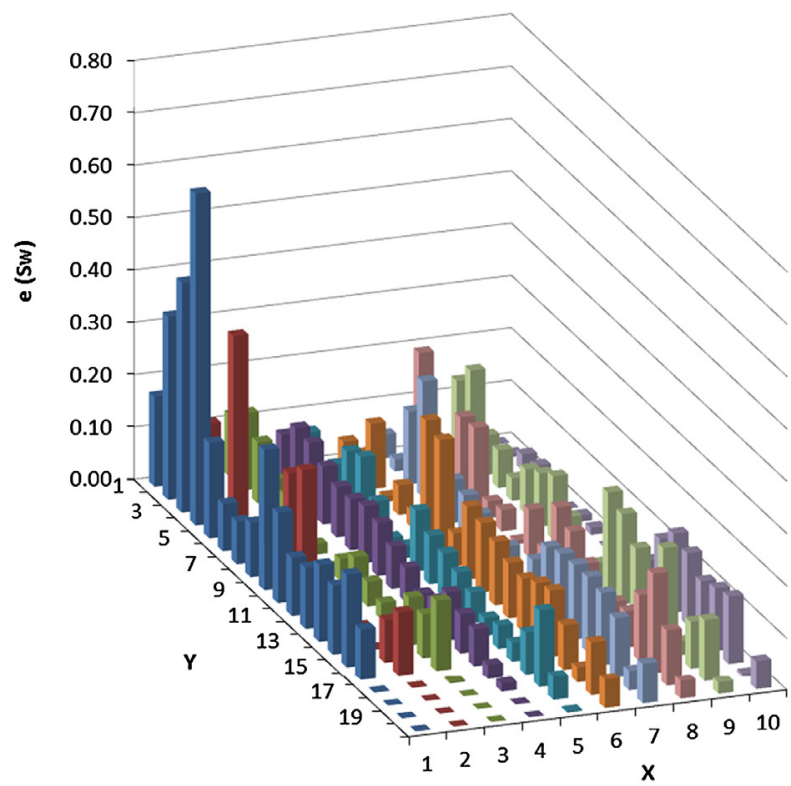

(b)

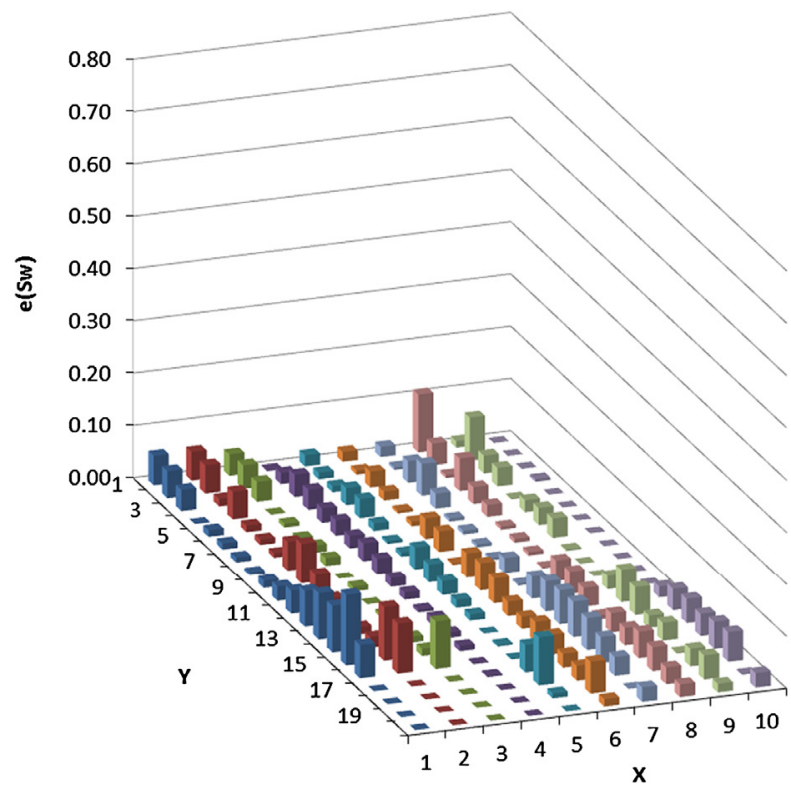

(d)

Fig. 22. Water saturation error in each permeability region of Test IV domain at 0.4 pore volumes injected for: (a) NCVFE for mesh of element length of $36 \mathrm{~m}$, (b) NCVFE for mesh of element length of $12 \mathrm{~m}$, (c) ICVFE for mesh of element length of $36 \mathrm{~m}$, (d) ICVFE for mesh of element length of $12 \mathrm{~m}$.

volumes centred around these interfaces. It uses a combination of the mixed hybrid finite element and CVFE methods. The main advantage of ICVFE is that an interface control volume encompasses, at most, two elements, which decreases the unphysical flow between neighbouring elements observed on other CVFE methods such as NCVFE. To verify this, we conducted a numerical study on the ICVFE method and compared its solutions to NCVFE. The ICVFE method outperformed the NCVFE method on all the tests representing highly heterogeneous and naturally fractured reservoirs. The ICVFE produces less unphysical flow between the different material properties of the systems and models more accurate fluid saturation profiles. Moreover, the ICVFE method defines the primary variables (pressure and saturation) on the interfaces of elements. Therefore, it computes a high resolution of the primary variables over the finite element mesh (the number of interfaces is larger than the number of elements). This down-scaling is attractive and convenient since the truncated numerical errors decrease with an increase in the degrees of freedom, showing - at best - linear convergence. In this paper we assume 


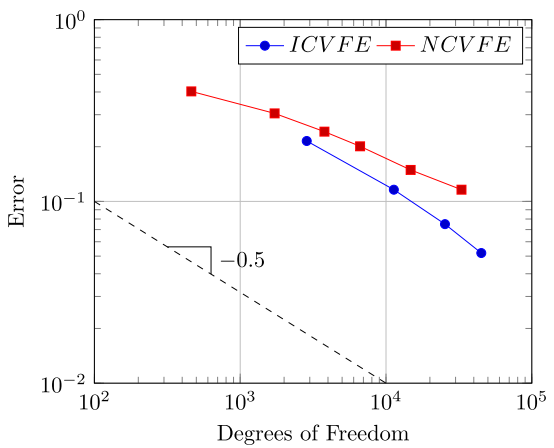

Fig. 23. Comparison of error in water saturation for Test IV domain between ICVFE and NCVFE methods at 0.4 pore volumes injected versus the meshes degrees of freedom. The ICVFE method produces less error than NCVFE.

gravity and capillarity are negligible, although this is not a constraint of the method. These forces, more complex domains, and other finite element shapes are the topic of future work.

\section{Acknowledgements}

This work was supported by Qatar Foundation - Research and Development, Qatar Science Leadership Program, and Qatar Carbonates and Carbon Storage Research Centre. We thank the Applied Modelling and Computation Group (AMCG) of Imperial College London for their help, advice and guidance.

\section{References}

[1] International Energy Agency, World energy outlook 2006, Tech. rep., OECD/IEA, Paris, 2007.

[2] R. Eymard, T. Gallouet, Hybrid finite element techniques for oil recovery simulation, Comput. Methods Appl. Mech. Eng. 74 (1989) 83-98.

[3] L. Fung, A. Hieben, L. Nshiera, Reservoir simulation with a control volume finite-element method, SPE Reserv. Eng. 7 (1991) 349-357.

[4] L.J. Durlofsky, A triangle based mixed finite-element-finite volume technique for modelling two phase flow through porous media, J. Comput. Phys. 105 (1993) 252-266.

[5] R. Huber, R. Helmig, Multi-phase flow in heterogeneous porous media: a classical finite element method versus an implicit pressure-explicit saturationbased mixed finite element-finite volume approach, Int. J. Numer. Methods Fluids 29 (1999) 899-920.

[6] S. Geiger, S. Roberts, S. Matthai, C. Zoppou, A. Burri, Combining finite element and finite volume methods for efficient multiphase flow simulation in highly heterogenous and structurally complex geological media, Geofluids 4 (2004) 284-299.

[7] K. Schmid, S. Geiger, K. Sorbie, Higher order fe-fv method on unstructured grids for transport and two-phase flow with variable viscosity in heterogeneous porous media, J. Comput. Phys. 241 (2013) 416-444.

[8] J.M. Thomas, D. Trujillo, Mixed finite volume methods, Int. J. Numer. Methods Eng. 46 (1999) 1351-1366.

[9] H. Nick, S. Matthai, A hybrid finite-element finite-volume method with embedded discontinuities for solute transport in heterogenous media, Vadose Zone J. 10 (2011) 299-312.

[10] A. Abushaikha, Numerical methods for modelling fluid flow in highly heterogeneous and fractured reservoirs, PhD thesis, Imperial College London, London, United Kingdom, 2013.

[11] M.G. Edwards, Higher-resolution hyperbolic-coupled-elliptic flux-continuous cvd schemes on structured and unstructured grids in 2-d, Int. J. Numer. Methods Fluids 51 (2006) 1059-1077.

[12] S. Bazr-Afkan, S. Matthai, A new hybrid simulation method for multiphase flow on unstructured grids with discrete representations of material interfaces, in: IAMG, 2011.

[13] Y. Zhang, M. King, A. Datta-Gupta, Robust streamline tracing using inter-cell fluxes in locally refined and unstructured grids, Water Resour. Res. (2012) 48.

[14] S. Verma, K.A. Aziz, Control volume scheme for flexible grids in reservoir simulation, in: The 1997 SPE Symposium on Reservoir Simulation, Dallas, 1997.

[15] M.G. Edwards, Higher-resolution hyperbolic-coupled-elliptic flux-continuous cvd schemes on structured and unstructured grids in 3-d, Int. J. Numer. Methods Fluids 51 (2006) 1079-1095.

[16] G. Chavent, J. Jaffre, Mathematical Models and Finite Elements for Reservoir Simulation, North-Holland, Amsterdam, 1986.

[17] L. Bergamaschi, S. Mantica, G. Manzini, A mixed finite element-finite volume formulation of the black-oil model, J. Sci. Comput. 20 (1989) 970-997.

[18] F. Berzzi, M. Fortin, Mixed and Hybrid Finite Element Methods, Springer, Berlin, 1991.

[19] L. Durlofsky, Accuracy of mixed and control volume finite element approximations to Darcy velocity and related quantities, Water Resour. Res. 30 (1994) 965-973.

[20] H. Hoteit, A. Firoozabadi, Compositional modelling by the combined discontinuous Galerkin and mixed methods, SPE J. 11 (2006) 19-34.

[21] C. Cordes, W. Kinzelbach, Comment on "Application of the mixed hybrid finite element approximation in a groundwater flow model: Luxury or necessity?” by R. Mosé, P. Siegel, P. Ackerer, and G. Chavent, Water Resour. Res. 32 (1996) 1905-1909.

[22] P. Lachassagne, E. Ledoux, G. deMarsily, Evaluation of hydrogeological parameters in heterogeneous porous media, in: Proceedings of Benidorm Symposium on Groundwater Management: Quantity and Quality, in: IAHS Publ., vol. 188, 1989, pp. 3-18.

[23] P. Mose, P. Siegel, P. Ackerer, G. Chavent, Application of the mixed hybrid finite element approximation in a groundwater flow model: luxury or necessity?, Water Resour. Res. 30 (1994) 3001-3012.

[24] G. Chavent, J. Roberts, A unified physical presentation of mixed, mixed-hybrid finite elements and standard finite difference approximations for the determination of velocities in water flow problems, Adv. Water Resour. 14 (1991) 329-348.

[25] A. Younes, R. Mose, P. Ackerer, G. Chavent, A new formulation of mixed finite element method for solving elliptic and parabolic pde with triangular elements, J. Comput. Phys. 149 (1999) 148-167. 
[26] P.A. Raviart, J.M. Thomas, A mixed finite element method for second order elliptic problems, in: Lect. Notes Math., vol. 606, 1977, pp. 292-315.

[27] R. Courant, Variational methods for the solution of problems of equilibrium and vibrations, Bull. Am. Math. Soc. 49 (1943) 1-23.

[28] V.R. Voller, Basic Control Volume Finite Element Methods for Fluids and Solids, World Scientific Publishing Co. Pte. Ltd., Singapore, 2009.

[29] J. Bear, Dynamics of Fluids in Porous Media, Dover, New York, 1972.

[30] A.T. Corey, The interrelation between gas and oil relative permeabilities, Prod. Mon. 19 (1954) 38-41.

[31] E.F. Kaasschieter, A.J.M. Huijben, Mixed-hybrid finite elements and streamline computation for the potential flow problem, Numer. Methods Partial Differ. Equ. 8 (1992) 221-266.

[32] A. Younes, P. Ackerer, G. Chavent, From mixed finite elements to finite volumes for elliptic pdes in two and three dimensions, Int. J. Numer. Methods Eng. 59 (2004) 365-388.

[33] C. Geuzaine, J. Remacle, Gmsh: a three-dimensional finite element mesh generator with built-in pre- and post-processing facilities, Int. J. Numer. Methods Eng. 79 (2009) 1309-1331.

[34] S. Buckley, M. Leverett, Mechanism of fluid displacements in sands, Trans. AIME 146 (1942) 107-116.

[35] M. Blunt, B. Rubin, Implicit flux limiting schemes for petroleum reservoir simulation, J. Comput. Phys. 102 (1992) 194-210.

[36] H. Hoteit, A. Firoozabadi, Numerical modeling of two-phase flow in heterogeneous permeable media with different capillarity pressures, Adv. Water Resour. 31 (2008) 56-73.

[37] L. Janicke, A. Kost, Error estimation and adaptive mesh generation in the 2d and 3d finite element method, IEEE Trans. Magn. 32 (1996) $1334-1337$.

[38] A. Abushaikha, O. Gosselin, Matrix-fracture transfer function in dual-medium flow simulation: review, comparison, and validation, in: Proceedings of Europec/EAGE Conference and Exhibition, Rome, 9-12 June, 2008, SPE-113890.

[39] L. Weixuan, L. Guang, Z. Dongxiao, An adaptive ANOVA-based PCKF for high-dimensional nonlinear inverse modeling, J. Comput. Phys. 258 (2014) $752-772$.

[40] Schlumberger, Eclipse reference manual 2011.2, Tech. rep., Schlumberger, Houston, 2011.

[41] N. Zhang, J. Yao, Z. Huang, Y. Wang, Accurate multiscale finite element method for numerical simulation of two-phase flow in fractured media using discrete-fracture model, J. Comput. Phys. 242 (2013) 420-438. 